\title{
The CMS barrel calorimeter response to particle beams from 2 to $350 \mathrm{GeV} / \mathrm{c}$
}

\section{CMS HCAL/ECAL Collaborations}

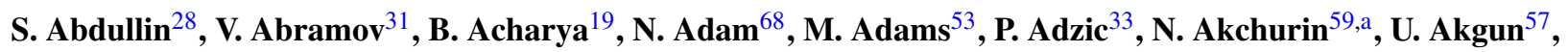
E. Albayrak ${ }^{57}$, R. Alemany-Fernandez ${ }^{25}$, N. Almeida ${ }^{25}$, G. Anagnostou ${ }^{12, b}$, D. Andelin ${ }^{52}$, E.W. Anderson ${ }^{48}$, M. Anfreville ${ }^{9}$, I. Anicin ${ }^{32,33}$, G. Antchev ${ }^{50}$, Z. Antunovic ${ }^{6}$, R. Arcidiacono ${ }^{23}$, M.W. Arenton ${ }^{52}$, E. Auffray ${ }^{34}$,

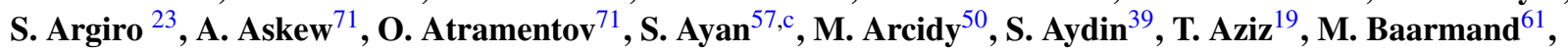
K. Babich ${ }^{26}$, S. Baccaro ${ }^{22, d}$, D. Baden ${ }^{54}$, S. Baffioni ${ }^{10}$, M.N. Bakirci ${ }^{39}$, M. Balazs ${ }^{52}$, Sud. Banerjee ${ }^{19}$, Sun. Banerjee ${ }^{19}$, R. Bard ${ }^{53}$, D. Barge ${ }^{55}$, V. Barnes ${ }^{72}$, D. Barney ${ }^{34}$, L. Barone ${ }^{22}$, A. Bartoloni ${ }^{22}$, C. Baty ${ }^{11}$, H. Bawa ${ }^{16}$, G. Baiatian ${ }^{1}$, D. Bandurin ${ }^{60}$, S. Beauceron ${ }^{34}$, K.W. Bell ${ }^{45}$, G. Bencze ${ }^{14}$, R. Benetta ${ }^{34}$, M. Bercher ${ }^{10}$, S. Beri $^{16}$, C. Bernet ${ }^{10}$, L. Berntzon ${ }^{59}$, U. Berthon ${ }^{10}$, M. Besancon ${ }^{9}$, B. Betev ${ }^{36}$, R. Beuselinck ${ }^{46}$, V. Bhatnagar ${ }^{16}$, A. Bhatti ${ }^{64}$,

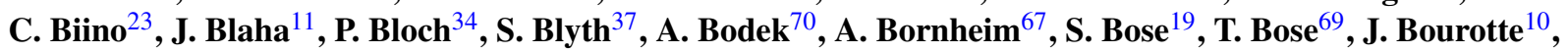
A.M. Brett ${ }^{36}$, R.M. Brown ${ }^{45}$, D. Britton ${ }^{46}$, H. Budd ${ }^{70}$, M. Buehler ${ }^{52}$, K. Burchesky ${ }^{53}$, P. Busson ${ }^{10}$, B. Camanzi $^{45}$,

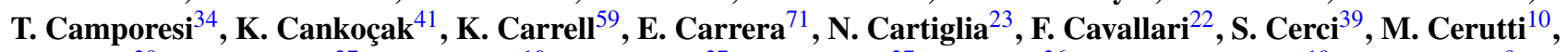
P. Chang ${ }^{38}$, Y.H. Chang ${ }^{37}$, C. Charlot ${ }^{10}$, E.A. Chen $^{37}$, W.T. Chen ${ }^{37}$, Z. Chen ${ }^{36}$, S. Chendvankar ${ }^{19}$, R. Chipaux ${ }^{9}$, B.C. Choudhary ${ }^{17}$, R.K. Choudhury ${ }^{18}$, Y. Chung ${ }^{70}$, W. Clarida ${ }^{57}$, D.J.A. Cockerill ${ }^{45}$, C. Combaret ${ }^{11}$, S. Conetti ${ }^{52}$, F. Cossutti ${ }^{24}$, B. Cox $^{52}$, L. Cremaldi ${ }^{66}$, P. Cushman ${ }^{63}$, D.G. Cussans ${ }^{44}$, I. Dafinei ${ }^{22}$, J. Damgov ${ }^{3,49}$,

D.R. Da Silva Di Calafiori ${ }^{36}$, G. Daskalakis ${ }^{12}$, G. Davatz ${ }^{36}$, A. David ${ }^{25}$, P. de Barbaro ${ }^{70}$, P. Debbins ${ }^{57}$, K. Deiters ${ }^{35}$, M. Dejardin ${ }^{9}$, M. Djordjevic ${ }^{33}$, M. Deliomeroglu ${ }^{41}$, R. Della Negra ${ }^{11}$, G. Della Ricca ${ }^{24}$, D. Del Re ${ }^{22}$, A. Demianov ${ }^{30}$,

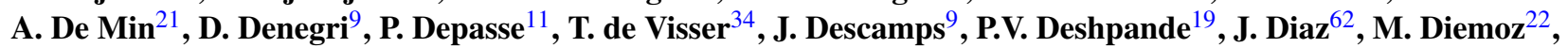
E. Di Marco ${ }^{22}$, L. Dimitrov ${ }^{3}$, G. Dissertori ${ }^{36}$, M. Dittmar ${ }^{36}$, L. Djambazov ${ }^{36}$, L. Dobrzynski ${ }^{10}$, S. Drndarevic ${ }^{32}$, J.E. Duboscq ${ }^{58}$, S. Dugad ${ }^{19}$, I. Dumanoglu ${ }^{39}$, F. Duru ${ }^{57}$, D. Dutta ${ }^{18}$, M. Dzelalija ${ }^{6}$, I. Efthymiopoulos ${ }^{34}$, J. Elias ${ }^{49}$, A. Elliott-Peisert ${ }^{34}$, H. El Mamouni ${ }^{11}$, D. Elvira ${ }^{49}$, I. Emeliantchik ${ }^{2}$, S. Eno $^{54}$, A. Ershov $^{30}$, S. Erturk $^{39, \mathrm{e}}$, S. Esen $^{69}$, ${\text { E. } \text { Eskut }^{39} \text {, I. Evangelou }}^{13}$, D.L. Evans ${ }^{44}$, B. Fabbro ${ }^{9}$, J.L. Faure ${ }^{9}$, J. Fay ${ }^{11}$, A. Fenyvesi ${ }^{15}$, F. Ferri ${ }^{9}$, W. Fisher ${ }^{68}$, P.S. Flower ${ }^{45}$, D. Franci ${ }^{22}$, G. Franzoni ${ }^{63}$, J. Freeman ${ }^{49}$, K. Freudenreich ${ }^{36}$, W. Funk ${ }^{34}$, S. Ganjour ${ }^{9}$, C. Gargiulo ${ }^{22}$, S. Gascon ${ }^{11}$, M. Gataullin ${ }^{67}$, V. Gaultney ${ }^{62}$, H. Gamsizkan ${ }^{40}$, V. Gavrilov ${ }^{28}$, Y. Geerebaert ${ }^{10}$, V. Genchev ${ }^{3}$, F.X. Gentit ${ }^{9}$, D. Gerbaudo ${ }^{68}$, Y. Gershtein ${ }^{71}$, A. Ghezzi ${ }^{34}$, M.D. Ghodgaonkar ${ }^{18}$, J. Gilly ${ }^{10}$, A. Givernaud ${ }^{9}$, S. Gleyzer ${ }^{71}$, S. Gninenko ${ }^{27}$, A. Go ${ }^{37}$, B. Gobbo ${ }^{24}$, N. Godinovic ${ }^{5}$, N. Golubev ${ }^{27}$, I. Golutvin ${ }^{26}$, P. Goncharov ${ }^{31}$, D. Gong ${ }^{63, \text { f, P. Govoni }}{ }^{21}$, N. Grant ${ }^{44}$, P. Gras ${ }^{9}$, T. Grassi ${ }^{54}$, D. Green ${ }^{49}$, R.J.S. Greenhalgh ${ }^{45}$, A. Gribushin ${ }^{30}$, B. Grinev ${ }^{43}$, L. Guevara Riveros ${ }^{10}$, J.P. Guillaud ${ }^{8}$, A. Gurtu ${ }^{19}$, A. Murat Güler ${ }^{40}$, E. Gülmez ${ }^{41}$, K. Gümüiş ${ }^{59}$,

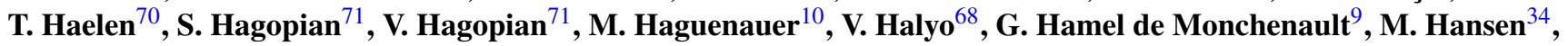
M. Hashemi ${ }^{20}$, J. Hauptman ${ }^{48}$, E. Hazen ${ }^{50}$, H.F. Heath ${ }^{44}$, A. Heering ${ }^{63}$, A. Heister ${ }^{50}$, B. Heltsley ${ }^{58}$, J.A. Hill ${ }^{45}$, W. Hintz ${ }^{36}$, R. Hirosky ${ }^{52}$, P.R. Hobson ${ }^{47}$, A. Honma ${ }^{34}$, G.W.S. Hou ${ }^{38}$, Y. Hsiung ${ }^{38}$, A. Hunt ${ }^{68}$, M. Husejko ${ }^{25}$,

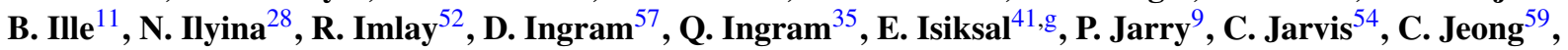
C. Jessop ${ }^{65}$, K. Johnson ${ }^{71}$, J. Jones ${ }^{68}$, D. Jovanovic ${ }^{32,33}$, K. Kaadze ${ }^{60}$, V. Kachanov ${ }^{31}$, V. Kaftanov ${ }^{28, h}$, S. Kailas ${ }^{18}$, V. Kalagin ${ }^{26}$, A. Kalinin ${ }^{31}$, S. Kalmani ${ }^{19}$, D. Karmgard ${ }^{65}$, S.K. Kataria ${ }^{18}$, M. Kaur ${ }^{16}$, M. Kaya ${ }^{41, i}$, O. Kaya ${ }^{41, i}$, A. Kayis-Topaksu ${ }^{39}$, R. Kellogg ${ }^{54}$, B.W. Kennedy ${ }^{45}$, A. Khmelnikov ${ }^{31}$, H. Kim ${ }^{59}$, I. Kisselevich ${ }^{28}$, K. Kloukinas ${ }^{34}$, O. Kodolova ${ }^{30}$, J. Kohli ${ }^{16}$, P. Kokkas ${ }^{13}$, T. Kolberg ${ }^{65}$, V. Kolossov ${ }^{28}$, A. Korablev ${ }^{31}$, Y. Korneev ${ }^{31}$, I. Kosarev ${ }^{26}$, L. Kramer ${ }^{62}$, N. Krasnikov ${ }^{27}$, A. Krinitsyn ${ }^{31}$, A. Krokhotin ${ }^{28}$, D. Krpic ${ }^{32}$, V. Kryshkin ${ }^{31}$, Y. Kubota ${ }^{63}$, A. Kubrik ${ }^{55}$, S. Kuleshov ${ }^{28}$, A. Kumar $^{16}$, P. Kumar ${ }^{18}$, S. Kunori ${ }^{54}$, C.M. Kuo ${ }^{37}$, P. Kurt ${ }^{39}$, P. Kyberd ${ }^{47}$, A. Kyriakis ${ }^{12}$,

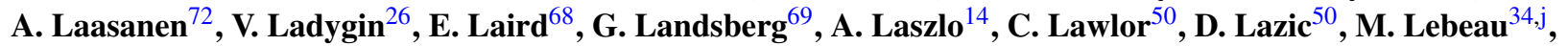
P. Lecomte ${ }^{36}$, P. Lecoq ${ }^{34}$, A. Ledovskoy ${ }^{52}$, S.-W. Lee ${ }^{59}$, G. Leshev ${ }^{36}$, M. Lethuillier ${ }^{11}$, L. Levchuk ${ }^{42}$, S.W. Lin ${ }^{38}$, W. Lin $^{37}$, S. Linn ${ }^{62}$, A.L. Lintern ${ }^{45}$, V. Litvine ${ }^{67}$, D. Litvintsev ${ }^{28}$, L. Litov ${ }^{4}$, L. Lobolo ${ }^{62}$, E. Locci ${ }^{9}$, A.B. Lodge ${ }^{45}$,

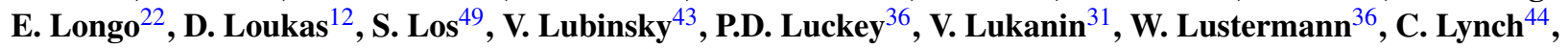
Y. Ma ${ }^{67}$, E. Machado ${ }^{50}$, H. Mahlke-Krueger ${ }^{58}$, M. Maity ${ }^{19}$, G. Majumder $^{19}$, M. Malberti ${ }^{21}$, J. Malclès ${ }^{9}$, D. Maletic ${ }^{33}$, I. Mandjavidze ${ }^{9}$, J. Mans ${ }^{68}$, N. Manthos $^{13}$, Y. Maravin ${ }^{60}$, C. Marchica ${ }^{35}$, N. Marinelli ${ }^{65}$, A. Markou ${ }^{12}$, 
C. Markou $^{12}$, D. Marlow ${ }^{68}$, P. Markowitz ${ }^{62}$, M. Marone ${ }^{23}$, G. Martinez ${ }^{62}$, H. Mathez ${ }^{11}$, V. Matveev ${ }^{27}$, C. Mavrommatis $^{12}$, G. Maurelli $^{11}$, K. Mazumdar ${ }^{19}$, P. Meridiani' ${ }^{34}$, J.P. Merlo ${ }^{57}$, H. Mermerkaya ${ }^{61}$, G. Mescheryakov ${ }^{26}$, A. Mestvirishvili ${ }^{57}$, V. Mikhailin ${ }^{30}$, P. Milenovic ${ }^{33}$, M. Miller ${ }^{57}$, G. Milleret ${ }^{10}$, P. Miné $^{10}$, A. Moeller ${ }^{57}$, M. Mohammadi-Najafabadi ${ }^{20}$, A.K. Mohanty ${ }^{18}$, P. Moissenz $^{26}$, N. Mondal $^{19}$, F. Moortgat $^{36}$, V. Mossolov ${ }^{2}$, M. Mur ${ }^{9}$, P. Musella ${ }^{25}$, Y. Musienko ${ }^{51}$, P. Nagaraj ${ }^{19}$, A. Nardulli ${ }^{36}$, J. Nash $^{46}$, P. Nedelec ${ }^{8}$, P. Negri ${ }^{21}$, H.B. Newman ${ }^{67}$, A. Nikitenko ${ }^{28}$, E. Norbeck $^{57}$, F. Nessi-Tedaldi ${ }^{36}$, M.M. Obertino ${ }^{23, \mathrm{k}}$, J. Olson ${ }^{57}$, Y. Onel ${ }^{57}$, G. Onengut $^{39}$, G. Organtini ${ }^{22}$, T. Orimoto ${ }^{67}$, C. Ozkan $^{40}$, H. Ozkurt ${ }^{39}$, S. Ozkorucuklu $^{41,1}$, F. Ozok ${ }^{57}$, M. Paganoni ${ }^{21}$, P. Paganini ${ }^{10}$, S. Paktinat ${ }^{20}$, A. Pal ${ }^{14}$, A. Palma ${ }^{22}$, B. Panev ${ }^{3}$, L. Pant ${ }^{18}$, A. Papadakis ${ }^{7}$,

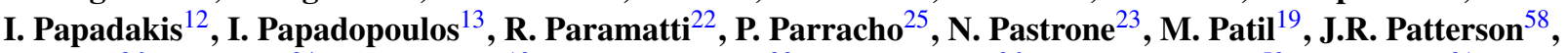
F. Pauss ${ }^{36}$, A. Penzo ${ }^{24}$, E. Petrakou ${ }^{12}$, S. Petrushanko ${ }^{30}$, A. Petrosyan ${ }^{26}$, D.G. Phillips II $^{52}$, V. Pikalov ${ }^{31}$,

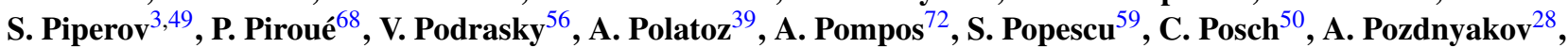
F. Ptochos ${ }^{7}$, I. Puljak ${ }^{5}$, A. Pullia ${ }^{21}$, T. Punz ${ }^{36}$, J. Puzovic ${ }^{32,33}$, W. Qian $^{53}$, S. Ragazzi ${ }^{21}$, S. Rahatlou ${ }^{22}$, R.M. Ralich ${ }^{61}$, J. Rande ${ }^{9}$, P.A. Razis ${ }^{7}$, N. Redaelli ${ }^{11}$, L. Reddy ${ }^{19}$, J. Reidy ${ }^{66}$, D. Renker ${ }^{35}$, S. Reucroft ${ }^{51}$, J.M. Reymond ${ }^{9}$,

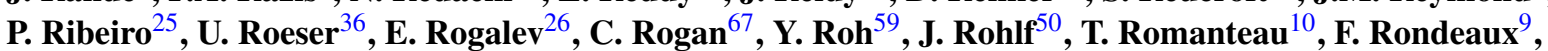
M. Ronquest ${ }^{52}$, A. Ronzhin $^{49}$, A. Rosowsky ${ }^{9}$, C. Rovelli $^{22}$, R. Ruchti ${ }^{65}$, P. Rumerio $^{34,54}$, R. Rusack ${ }^{63}$, S.V. Rusakov ${ }^{29}$, M.J. Ryan ${ }^{46}$, A. Ryazanov ${ }^{31}$, G. Safronov ${ }^{28}$, L. Sala ${ }^{21}$, R. Salerno ${ }^{21}$, D.A. Sanders ${ }^{66}$, F. Santanastasio ${ }^{22,54}$, C. Sanzeni ${ }^{56}$, L. Sarycheva ${ }^{30}$, B. Satyanarayana ${ }^{19}$, D. Schinzel ${ }^{36}$, I. Schmidt ${ }^{57}$, C. Seez ${ }^{46}$, S. Sekmen ${ }^{40}$, S. Semenov ${ }^{28}$, V. Senchishin ${ }^{43}$, S. Sergeyev ${ }^{49}$, M. Serin ${ }^{40}$, R. Sever ${ }^{40}$, P. Sharp ${ }^{34,46}$,

C.H. Shepherd-Themistocleous ${ }^{45}$, C. Siamitros ${ }^{47}$, D. Sillou ${ }^{8}$, J.B. Singh ${ }^{16}$, A. Singovsky ${ }^{63}$, Y. Sirois ${ }^{10}$, A. Sirunyan ${ }^{1}$, J. Silva ${ }^{25}$, P. Silva ${ }^{25}$, A. Skuja ${ }^{54}$, S. Sharma ${ }^{19}$, B. Sherwood ${ }^{63}$, J.G. Shiu ${ }^{38}$, R.K. Shivpuri ${ }^{17}$, P. Shukla ${ }^{18}$, N. Shumeiko ${ }^{2}$, V. Smirnov ${ }^{26}$, B.J. Smith ${ }^{45}$, V.J. Smith ${ }^{44}$, K. Sogut ${ }^{39, m}$, N. Sonmez ${ }^{41, n}$, P. Sorokin ${ }^{42}$, M. Spezziga ${ }^{59}$, M. Sproston ${ }^{45}$, R. Stefanovich ${ }^{2}$, F. Stöckli ${ }^{36}$, V. Stolin ${ }^{28}$, K. Sudhakar ${ }^{19}$, L. Sulak ${ }^{50}$, H. Suter ${ }^{36}$, I. Suzuki ${ }^{49}$, J. Swain ${ }^{51}$, T. Tabarelli de Fatis ${ }^{21}$, V. Talov ${ }^{31}$, M. Takahashi ${ }^{46}$, A. Tcheremoukhine ${ }^{26}$, O. Teller ${ }^{34}$, K. Teplov ${ }^{30}$,

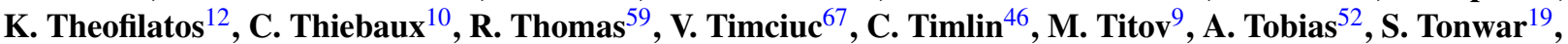

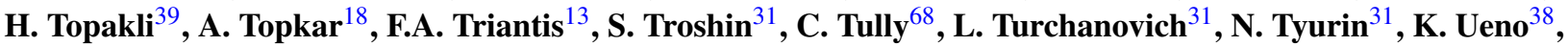

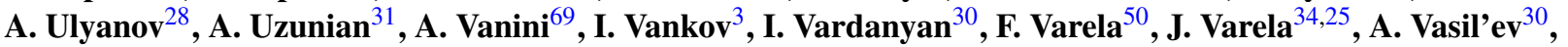
M. Velasco ${ }^{55}$, M. Vergili ${ }^{39}$, P. Verma ${ }^{19}$, P. Verrecchia ${ }^{9}$, G. Vesztergombi ${ }^{14}$, J. Veverka ${ }^{67}$, P. Vichoudis $^{34}$, R. Vidal ${ }^{49}$, T. Virdee ${ }^{34,46}$, A. Vishnevskiy ${ }^{26}$, E. Vlassov ${ }^{34,28}$, I. Vodopiyanov ${ }^{61}$, I. Volobouev $^{59}$, A. Volkov ${ }^{31}$, A. Volodko ${ }^{26}$, H.P. Von Gunten ${ }^{36}$, L. Wang ${ }^{54}$, M. Wang ${ }^{38}$, D. Wardrope ${ }^{46}$, M. Weber ${ }^{36}$, J. Weng ${ }^{36}$, J. Werner ${ }^{68}$, M. Wetstein ${ }^{54}$, D. Winn ${ }^{56}$, R. Wigmans ${ }^{59}$, J.H. Williams ${ }^{45}$, J. Whitmore ${ }^{49}$, S. Won ${ }^{55}$, S.X. Wu ${ }^{50}$, Y. Yang ${ }^{67}$, I. Yaselli ${ }^{47}$, E. Yazgan ${ }^{59}$, T. Yetkin ${ }^{57}$, R. Yohay ${ }^{52}$, A. Zabi ${ }^{10}$, P. Zalan ${ }^{14}$, N. Zamiatin ${ }^{26}$, A. Zarubin ${ }^{26}$, S. Zelepoukine ${ }^{31}$, M. Zeyrek ${ }^{40}$,

\section{J. Zhang ${ }^{63}$, L.Y. Zhang ${ }^{67}$, K. Zhu ${ }^{67}$}

${ }^{1}$ Yerevan Physics Institute, Yerevan, Armenia

${ }^{2}$ NCPHEP, Minsk, Belarus

${ }^{3}$ Institute for Nuclear Research and Nuclear Energy, Bulgarian Academy of Science, Sofia, Bulgaria

${ }_{5}^{4}$ Sofia University, Sofia, Bulgaria

${ }^{5}$ Technical University of Split, Split, Croatia

${ }^{6}$ University of Split, Split, Croatia

${ }^{7}$ University of Cyprus, Nicosia, Cyprus

${ }^{8}$ Laboratoire d'Annecy-le-Vieux de Physique des Particules, IN2P3-CNRS, Annecy-le-Vieux, France

${ }^{9}$ DSM/DAPNIA, CEA/Saclay, Gif-sur-Yvette, France

${ }^{10}$ Laboratoire Leprince-Ringuet, Ecole Polytechnique, IN2P3-CNRS, Palaiseau, France

${ }^{11}$ Institut de Physique Nucléaire de Lyon, Université Lyon 1, CNRS/IN2P3, Villeurbanne, France

${ }^{12}$ Institute of Nuclear Physics "Demokritos", Aghia Paraskevi, Greece

${ }^{13}$ University of Ioánnina, Ioánnina, Greece

${ }^{14}$ KFKI-RMKI, Research Institute for Particle and Nuclear Physics, Budapest, Hungary

${ }^{15}$ ATOMKI, Debrecen, Hungary

${ }^{16}$ Panjab University, Chandigarh, India

${ }^{17}$ University of Delhi, Delhi, India

${ }^{18}$ Bhabha Atomic Research Centre, Mumbai, India

${ }^{19}$ Tata Institute of Fundamental Research, Mumbai, India

${ }^{20}$ Institute for Studies in Theoretical Physics and Sharif University of Technology, Tehran, Iran

${ }^{21}$ Istituto Nazionale di Fisica Nucleare e Universita Degli Studi Milano Bicocca, Milano, Italy

${ }^{22}$ Università di Roma I e Sezione dell' INFN, Roma, Italy

${ }^{23}$ Università di Torino e Sezione dell' INFN, Torino, Italy

${ }^{24}$ Università di Trieste e Sezione dell' INFN, Trieste, Italy

${ }^{25}$ Laboratório de Instrumentação e Física Experimental de Partículas, Lisboa, Portugal 
${ }^{26}$ JINR, Dubna, Russia

${ }^{27}$ Institute for Nuclear Research, Moscow, Russia

${ }^{28}$ ITEP, Moscow, Russia

${ }^{29}$ Lebedev Physical Institute, Moscow, Russia

${ }^{30}$ Moscow State University, Moscow, Russia

${ }^{31}$ IHEP, Protvino, Russia

${ }^{32}$ University of Belgrade, Belgrade, Serbia

${ }^{33}$ Vinca Institute of Nuclear Sciences, Belgrade, Serbia

${ }^{34}$ CERN, European Organisation for Nuclear Research, Geneva, Switzerland

${ }^{35}$ Paul Scherrer Institut, Villigen, Switzerland

${ }^{36}$ Institute for Particle Physics, ETH Zurich, Zurich, Switzerland

${ }^{37}$ National Central University, Chung-Li, Taiwan

${ }^{38}$ National Taiwan University (NTU), Taipei, Taiwan

${ }^{39}$ Çukurova University, Adana, Turkey

${ }^{40}$ Middle East Technical University, Ankara, Turkey

${ }^{41}$ Boğaziçi University, Istanbul, Turkey

${ }^{42}$ KIPT, Kharkov, Ukraine

${ }^{43}$ Single Crystal Institute, Kharkov, Ukraine

${ }^{44}$ University of Bristol, Bristol, UK

${ }^{45}$ Rutherford Appleton Laboratory, Didcot, UK

${ }^{46}$ Imperial College, University of London, London, UK

${ }^{47}$ Brunel University, Uxbridge, UK

${ }^{48}$ Iowa State University, Ames, IA, USA

${ }^{49}$ Fermi National Accelerator Laboratory, Batavia, IL, USA

${ }^{50}$ Boston University, Boston, MA, USA

${ }^{51}$ Northeastern University, Boston, MA, USA

${ }^{52}$ University of Virginia, Charlottesville, VA, USA

${ }^{53}$ University of Illinois at Chicago, Chicago, IL, USA

${ }^{54}$ University of Maryland, College Park, MD, USA

${ }^{55}$ Northwestern University, Evanston, IL, USA

${ }^{56}$ Fairfield University, Fairfield, CT, USA

${ }^{57}$ University of Iowa, Iowa City, IA, USA

${ }^{58}$ Cornell University, Ithaca, NY, USA

${ }^{59}$ Texas Tech University, Lubbock, TX, USA

${ }^{60}$ Kansas State University, Manhattan, KS, USA

${ }^{61}$ Florida Institute of Technology, Melbourne, FL, USA

${ }^{62}$ Florida International University, Miami, FL, USA

${ }^{63}$ University of Minnesota, Minneapolis, MN, USA

${ }^{64}$ Rockefeller University, New York, NY, USA

${ }^{65}$ University of Notre Dame, Notre Dame, IN, USA

${ }^{66}$ University of Mississippi, Oxford, MS, USA

${ }^{67}$ California Institute of Technology, Pasadena, CA, USA

${ }^{68}$ Princeton University, Princeton, NJ, USA

${ }^{69}$ Brown University, Providence, RI, USA

${ }^{70}$ University of Rochester, Rochester, NY, USA

${ }^{71}$ Florida State University, Tallahassee, FL, USA

${ }^{72}$ Purdue University, West Lafayette, IN, USA

Received: 29 June 2008 / Revised: 24 November 2008 / Published online: 3 March 2009

(C) Springer-Verlag / Società Italiana di Fisica 2009

Abstract The response of the CMS barrel calorimeter (electromagnetic plus hadronic) to hadrons, electrons and muons over a wide momentum range from 2 to $350 \mathrm{GeV} / c$ has been measured. To our knowledge, this is the widest range of momenta in which any calorimeter system has been studied. These tests, carried out at the $\mathrm{H} 2$ beam-line at CERN, provide a wealth of information, especially at low energies. The analysis of the differences in calorimeter response to charged pions, kaons, protons and antiprotons and a detailed discussion of the underlying phenomena are presented. We also show techniques that apply corrections to the signals from the considerably different electromagnetic (EB) and hadronic (HB) barrel calorimeters in reconstructing the energies of hadrons. Above $5 \mathrm{GeV} / c$, these corrections improve the energy resolution of the combined system where the stochastic term equals $84.7 \pm 1.6 \%$ and the constant term is $7.4 \pm 0.8 \%$. The corrected mean response remains constant within $1.3 \%$ rms. 


\section{Introduction}

The CMS calorimeters have distinct hadronic (HCAL) and electromagnetic (ECAL) systems. The central HCAL is made of brass and scintillators [1] while the ECAL comprises lead tungstate crystals $\left(\mathrm{PbWO}_{4}\right)$ [2]. The calorimeters are divided into the barrel (HB and $\mathrm{EB}$ ) and the endcap (HE, EE and pre-shower, ES) sections inside a cryostat of $5.9 \mathrm{~m}$ inner diameter, containing a superconducting solenoidal coil providing a 4-T magnetic field. The HB design maximizes the number of interaction lengths $\left(\lambda_{I}\right)$ inside the cryostat and is limited to $5.8 \lambda_{\mathrm{I}}$ at $\eta=0$. The EB adds 1.1 $\lambda_{\mathrm{I}}$ and the material between EB and HB adds another $0.1 \lambda_{\mathrm{I}}$. The outer hadron calorimeter (HO) was constructed to sample the energy leakage outside of the cryostat [3]. There are also two forward calorimeters (HF) made of iron and quartz fibers [4]. Figure 1.1 shows a schematic of the calorimeters inside and around the magnet.

This paper reports on the barrel calorimeter ( $\mathrm{HB}, \mathrm{HO}$ and EB) responses to beam particles. The measurements

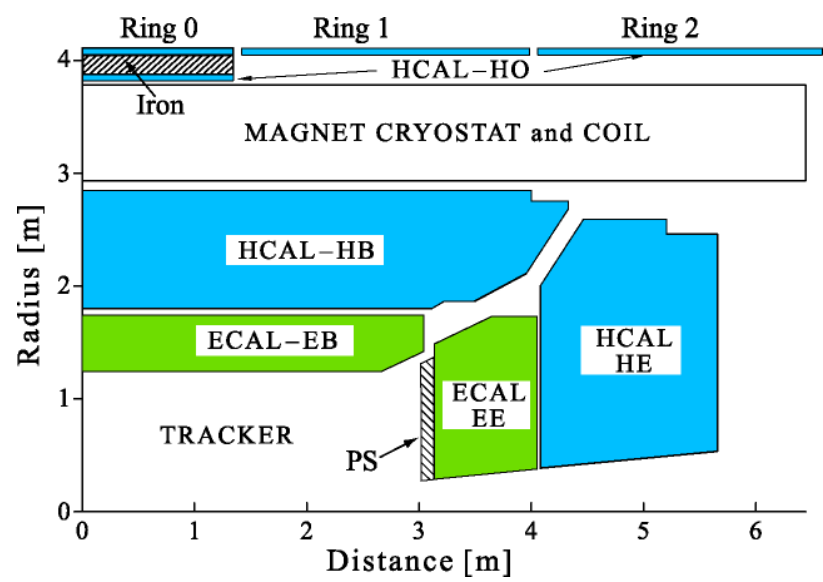

Fig. 1.1 Location of the ECAL and the HCAL detectors (quarter slice-longitudinal cross section) in and around the CMS magnet

a e-mail: nural.akchurin@ttu.edu

bow at: RWTH, I. Physikalisches Institut, Aachen, Germany.

${ }^{\mathrm{c}}$ Now at: University of Pennsylvania, Philladelphia, PA, USA.

${ }^{\mathrm{d}}$ Now at: ENEA, Casaccia Research Center, S. Maria di Galeria, Italy. eAt: Niğde University, Niğde, Turkey.

${ }^{\mathrm{f}}$ Now at: Southern Methodist University, Dallas, TX, USA.

${ }^{\mathrm{g}}$ At: Marmara University, Istanbul, Turkey.

${ }^{\mathrm{h}}$ Deceased.

${ }^{\mathrm{i}}$ At: Kafkas University, Kars, Turkey.

${ }^{\mathrm{j}}$ Now at: University of Athens, Greece.

${ }^{k}$ Now at: Università del Piemonte Orientale, Novara, Italy.

${ }^{1}$ At: Süleyman Demirel University, Isparta, Turkey.

${ }^{m}$ At: Mersin University, Mersin, Turkey.

${ }^{\mathrm{n}}$ At: Izmir Yüksek Teknoloji Enstitüsü, Izmir, Turkey. were performed with production modules and front-end electronics as in the final CMS detector configuration. A special beam line was constructed to measure the calorimeter response down to $2 \mathrm{GeV} / c$. This was necessary since a large fraction of the particles reaching the CMS calorimeters in the LHC will have energies below $20 \mathrm{GeV}$.

\subsection{HCAL barrel (HB) calorimeter}

The $\mathrm{HB}$ and $\mathrm{HO}$ are designed to measure the timing, angular direction and energy of hadronic showers. These quantities are needed for the calorimetric triggers and online reconstruction of jets and missing transverse energy. The cylindrically symmetric HB surrounds the EB. It consists of alternating layers of brass and plastic scintillator tiles (3.7 mm SCSN81 from Kuraray except the innermost layer $1.0 \mathrm{~cm}$ BC408 from Bicron). The HB design necessarily requires minimizing the amount of space devoted to the active medium. The scintillator tiles are read out with embedded wavelength shifting (WLS) fibers. Brass was chosen as the absorber material because it is nonmagnetic and cost is affordable. This design makes construction relatively simple, lends itself to projective tower geometry, and eliminates uninstrumented gaps. The brass type is $\mathrm{C} 26000$ (cartridge brass) and is composed of $70 \% \mathrm{Cu}$ and $30 \% \mathrm{Zn}$. The brass density is $8.83 \mathrm{~g} / \mathrm{cm}^{3}$ with interaction length $\lambda_{I}=16.4 \mathrm{~cm}$ and radiation length $X_{0}=1.5 \mathrm{~cm}$.

The HB covers the pseudorapidity range $|\eta|<1.3$ and consists of 36 identical azimuthal wedges $\left(\Delta \phi=20^{\circ}\right)$ which form two half-barrels ( $\mathrm{HB}+$ and $\mathrm{HB}-$ ). Each halfbarrel is inserted from either end of the cryostat and positioned around the central axis of the CMS magnet. Each wedge is further segmented into four azimuthal $\left(\Delta \phi=5^{\circ}\right)$ sectors. The plates are bolted together in a staggered geometry resulting in a configuration that contains no projective passive material for the full radial extent of a wedge. The innermost and outermost plates are made of stainless steel to provide structural strength. The scintillator is divided into $16 \eta$ sectors, resulting in a tower segmentation of $(\Delta \eta, \Delta \phi)=(0.087,0.087)$.

Each wedge contains 72 channels of front-end electronics mounted on the detector periphery near $|\eta| \approx 1$.3. These circuits are housed in an enclosure referred to as a readout box (RBX). Each of these RBXs is further divided into four readout modules (RM). A single RM contains a 19-channel hybrid photodiode (HPD) that registers signals from $16 \eta$ towers of a single $\phi$ sector.

The HPD is a planar structure consisting of a photocathode and a silicon diode separated by $3.5 \mathrm{~mm}$ of vacuum. Photoelectrons are accelerated by a $\sim 8-\mathrm{kV}$ potential and strike the diode causing ionization which results in a gain of 1,600. The diode consists of 19 electrically independent 
readouts. The HPD signals are fed into three 6-channel readout cards located inside the RM, based on a custom ASIC which performs charge integration and encoding (QIE). The $r m s$ noise per tower is about $200 \mathrm{MeV}$. For the $\mathrm{HB}$, six time samples $(6 \times 25 \mathrm{~ns})$ from $3 \times 3 \mathrm{HB}$ towers were used for energy reconstruction. The signal is about $75 \mathrm{~ns}$ wide with a small tail extending another $50 \mathrm{~ns}$. The zero input response of the QIE (pedestals) are measured and subtracted for each run.

The QIE is a nonlinear multi-range ADC designed to provide approximately constant fractional precision over a wide dynamic range. This is accomplished with a floating-point analog-to-digital conversion in which the bin width in each of four ranges is increased in proportion to the input amplitude. In addition, the QIE has four time interleaved stages. The output of the QIE contains 2 bits of range (exponent) and 5 bits of mantissa. Details of the HB geometry, construction and electronics are reported elsewhere [5-8].

The design of the outer calorimeter (HO) scintillating tiles is similar to that of the HB. The scintillator plates are $1 \mathrm{~cm}$ thick BC408. The HO counters are grouped in six segments and the transverse segmentation is identical to that of the HB. Two layers of scintillating tiles are inserted in the central muon system while the other two rings have one layer of scintillating tiles (see Fig. 1.1). The readout and the electronics for $\mathrm{HO}$ are the same as in the $\mathrm{HB}$ system.

\subsection{ECAL barrel (EB) calorimeter}

The electromagnetic calorimeter used for these measurements consisted of a complete production EB Supermodule (SM) of width $\Delta \phi=20^{\circ}$ containing 1,700 crystals. The EB crystals are slightly tapered with front-face dimensions of $2.2 \times 2.2 \mathrm{~cm}^{2}$ and a crystal length of $23 \mathrm{~cm}$ or $25.8 X_{0}$ (see Sect. 4.3 for discussion on the interaction length). In order to avoid cracks in the barrel, the axes of the crystals are tilted by $3^{\circ}$ in both polar and azimuthal angle with respect to the direction of particle tracks originating from the interaction point. The EB covers a range $|\eta|<1.48$ and consists of 36 SMs containing 61,200 crystals.

The light emitted in the crystals is converted to an electrical signal using avalanche photodiodes (APD). Two APDs (Hamamatsu S8148) are glued to the back of each crystal. The rms electronics noise per crystal was found to be about $45 \mathrm{MeV}$. A minimum ionizing particle deposits about $250 \mathrm{MeV}$ over the full length of the crystal $[9,10]$.

The EB signal from the APD is amplified and shaped before being digitized by the ADC clocked at $40 \mathrm{MHz}$. The energy in the EB is computed as a weighted sum of several time samples of the waveform. The choice of weights and the number of samples are dictated by the desire to minimize the noise which is measured with no input signal (pedestal). In the test beam environment, the phase with respect to the
$40 \mathrm{MHz}$ clock is random. Even though the signal is about $200 \mathrm{~ns}$ wide, only six time samples $(6 \times 25 \mathrm{~ns})$ from $7 \times 7$ crystals were used for energy reconstruction with pedestal subtraction.

\section{Test beam setup}

The data were recorded during 2006 at the CERN H2 test beam. Figure 2.1 shows a photograph of the moving platform that held two production HB wedges plus a production EB SM which was placed in front of the $\mathrm{HB}$, and the HO behind the HB. The HE module seen on the platform was not used in this test. The placement of the components is in the same geometric relationship as in the CMS experiment. The two-dimensional movement of the platform in the $\phi$ and $\eta$ directions allowed the beam to be directed onto any tower of the calorimeter mimicking a particle trajectory from the interaction point of the CMS experiment. Four scintillation counters were located three meters upstream of the calorimeters and a coincidence between a subset of the counters was used as the trigger.

Temperature stability is critical for the ECAL as both the response of the crystals and the APDs change with temperature. The temperature was stabilized by enclosing the EB $\mathrm{SM}$ (except in the beam direction) in $5 \mathrm{~cm}$ aluminum plates with cooling water pipes embedded in the plates. The entire SM was wrapped with a thermal blanket and the temperature was stabilized at $21^{\circ} \mathrm{C}$ within $\pm 0.05^{\circ} \mathrm{C}$.

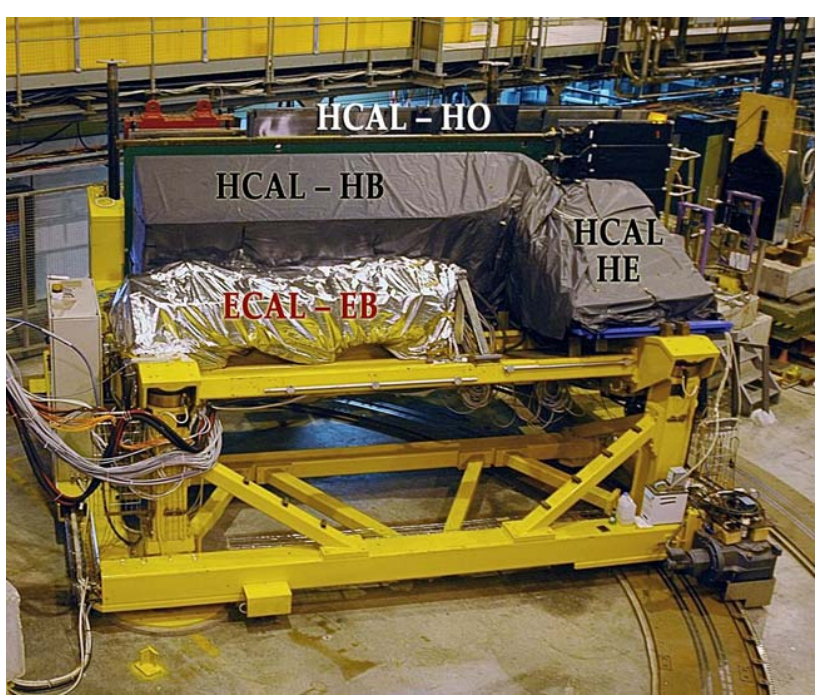

Fig. 2.1 The ECAL and the HCAL modules on a moving platform in the CERN H2 test beam area. The transporter table which supported the wedges is designed to move in $\phi$ and $\eta$ directions, approximately 0 to $30^{\circ}$ in $\phi$ and 0 to 3.0 in $\eta$ 


\subsection{H2 beam line and particle identification}

Because a tertiary beam was required to study low momentum $(<10 \mathrm{GeV} / c)$ particles, considerable effort was made to clean up the beams and perform particle identification. Figure 2.2 schematically depicts the CERN H2 beam line. The beam line is designed to operate in two distinct modes. In the high energy mode, various particles are produced when $450 \mathrm{GeV} / c$ protons from the Super Proton Synchrotron (SPS) strike a production target (T2) $590.9 \mathrm{~m}$ upstream of the calorimeters, and particle momenta range between 15 and $350 \mathrm{GeV} / c$. In the very low energy (VLE) mode, an additional target (T22) located $97.0 \mathrm{~m}$ upstream of the calorimeter is used for particle production and the momenta of particles are limited to $\leq 9 \mathrm{GeV} / c$. As shown in Fig. 2.2, a dog-leg configuration is utilized for the momentum selection of these low momentum particles.

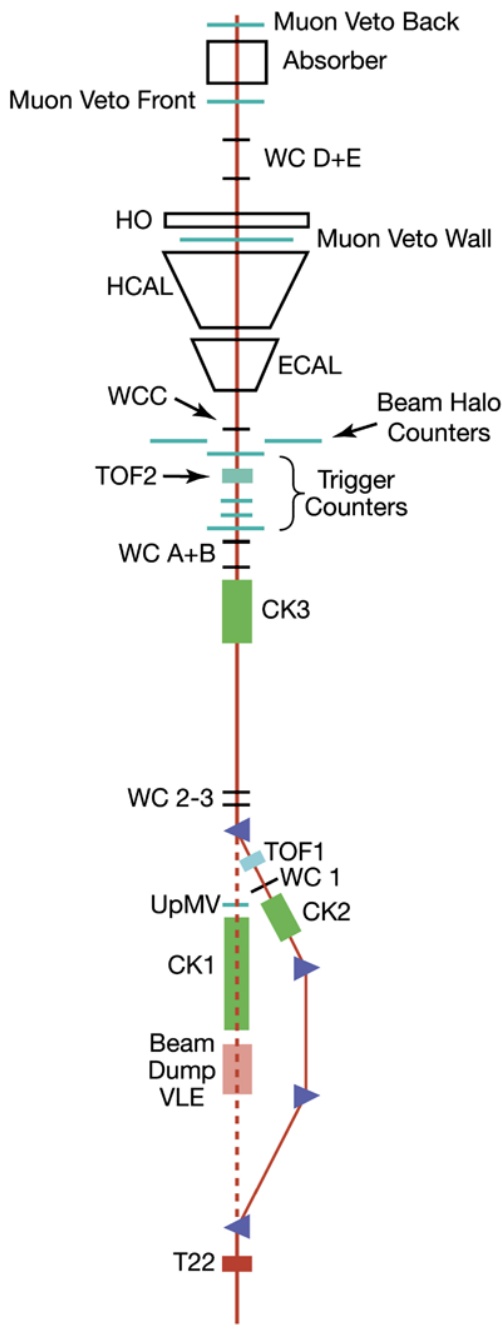

Fig. 2.2 The CERN H2 beam line and the experimental setup are shown schematically. In the VLE mode, the T22 target and a beam dump were inserted into the beam line, and the low energy particles were steered through the dog-leg
In the high energy mode, the T22 target and the VLE beam dump were removed from the beam line. The maximum usable beam momentum was $100 \mathrm{GeV} / c$ for electrons and $350 \mathrm{GeV} / c$ for hadrons. In the VLE mode, two Cherenkov counters (CK2 and CK3), two time-of-flight counters (TOF1 and TOF2) and muon counters (Muon Veto Wall (MVW) of $100 \times 226 \mathrm{~cm}^{2}$, Muon Veto Front (MVF) of $80 \times 80 \mathrm{~cm}^{2}$ and Muon Veto Back (MVB) of $80 \times 80 \mathrm{~cm}^{2}$ ) were used to positively tag electrons, pions, kaons, protons, antiprotons and muons.

CK2 is a $1.85-\mathrm{m}$ long Cherenkov counter filled with $\mathrm{CO}_{2}$ and was used to identify electrons in the VLE mode. At 0.35 bar, no other charged particles gave a signal and the counter was better than $99 \%$ efficient in identifying electrons. It produces 6 photoelectrons ( $p e$ ) for particle passage with $\beta=1$. CK3 is also $1.85-\mathrm{m}$ long and was filled with Freon134a. ${ }^{1}$ The pressure in CK3 was set depending on the desired discrimination between electrons, pions, and kaons. For example, at lower beam momenta $\left(P_{b} \leq 3 \mathrm{GeV} / c\right)$, it was set to tag electrons at 0.88 bar yielding $19 p e$ for $\beta=1$. At higher momenta $\left(P_{b}>4 \mathrm{GeV} / c\right), \mathrm{CK} 3$ was usually run at 1.2 bar in order to separate pions from kaons and protons where a $\beta=1$ particle yielded $25.5 \mathrm{pe}$.

Time-of-flight counters (TOF1 and TOF2) were separated by $\sim 55 \mathrm{~m}$. Each scintillator plate was $10 \times 10 \mathrm{~cm}^{2}$ in area and $2-\mathrm{cm}$ thick. Two trapezoidal air-core light guides on either side of the plate funneled the scintillation light to two fast photomultiplier tubes (Hamamatsu R5900). The analog pulses were discriminated by constant fraction discriminators. The time resolution obtained by this system was $\sim 300$ ps. Protons were well-separated from pions (and kaons) up to $7 \mathrm{GeV} / c$ with this time-of-flight system alone. Pions and kaons have $\pm 1 \sigma$ TOF overlap at momenta of $5.6 \mathrm{GeV} / c$, while kaons and protons overlap at $9.5 \mathrm{GeV} / c$. Figures 2.3 and 2.4 display the identified particles in 3 and $8 \mathrm{GeV} / c$ negative hadron beams.

Energetic muons were tagged with MVF and MVB counters as well as the MVW counters. MVF and MVB were large $\left(80 \times 80 \mathrm{~cm}^{2}\right)$ scintillation counters and were placed well behind the calorimeters. In order to absorb the soft beam component in the beam line, an $80-\mathrm{cm}$ thick iron block was inserted in front of MVB. When tested with a pure muon beam at $225 \mathrm{GeV} / c$, the efficiency of muon rejection was found to be better than $99 \%$. MVW consisted of 8 individual scintillation counters, each measuring $80 \times 100 \mathrm{~cm}^{2}$, placed closely behind the HB. These counters were positioned horizontally with a 2 -cm overlap between them, hence covering

\footnotetext{
${ }^{1}$ Freon $134 \mathrm{a}$ is an ozone-friendly gas. Based on the measurements during the beam test, we find Freon 134a's refractive index to be 1.00065 , which is also consistent with the estimates based on its molecular weight.
} 


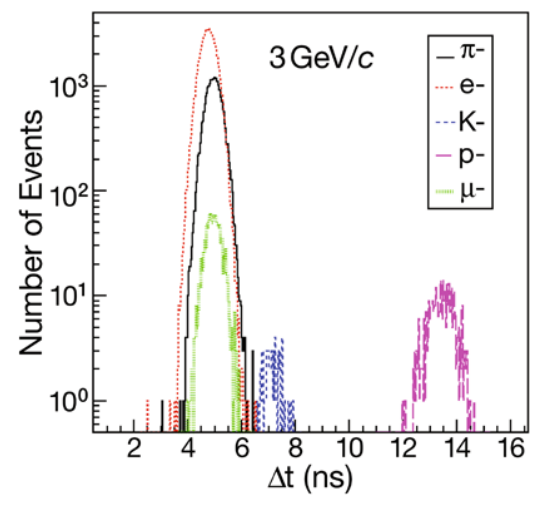

Fig. 2.3 (Color online) The particle identification was carried out with CK2 and CK3, TOF1 and TOF2, and MVW in the VLE mode. The distributions of the time of flight between TOF1 and TOF2 are shown for different particles

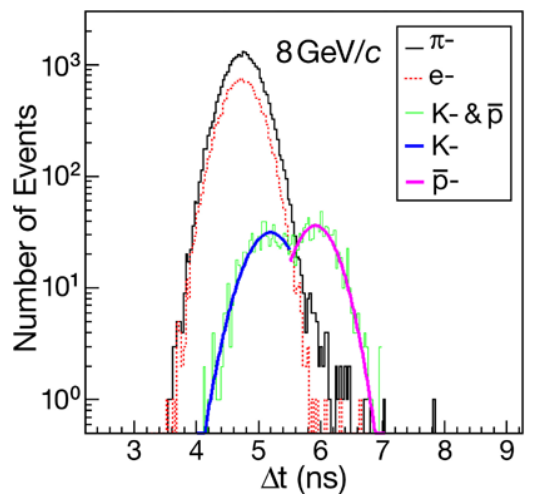

Fig. 2.4 (Color online) The same as Fig. 2.3 but for an $8 \mathrm{GeV} / c$ negative hadron beam. The solid blue and purple lines indicate fits to data (green histogram) for $K^{-}$and $\bar{p}$, respectively

a region of $226 \mathrm{~cm}$ in the vertical and $100 \mathrm{~cm}$ in the horizontal directions. In addition to tagging low momentum (2$5 \mathrm{GeV} / c$ ) beam muons, MVW was also used to study the details of late developing hadronic showers.

In addition to the aforementioned particle ID detectors, six delay-line chambers (WC1 through WC3 and WCA through WCC upstream of the EB+HB), four scintillation counters (S1 through S4) for triggering and four scintillation beam halo counters (BH1 through BH4) were used in the experiment. The spatial resolution afforded by the delay-line chambers was $\sim 350 \mu \mathrm{m}$ in both the $x$ - and $y$ coordinates. The beam trigger typically consisted of the coincidence S1.S2.S4 which defined a $4 \times 4 \mathrm{~cm}^{2}$ area on the front face of the calorimeter. The $\mathrm{S} 4$ counter pulse height was used to eliminate multi-particle events off-line since it gave a clean pulse height distribution for single and multiple particles in the beam (see Fig. 2.5). BH counters, each measuring $30 \times 100 \mathrm{~cm}^{2}$ in size, were arranged such that the beam passed through a $7 \times 7 \mathrm{~cm}^{2}$ opening. These counters were positioned $17 \mathrm{~cm}$ downstream of the last trigger scintillator $\mathrm{S} 4$ and were effective in vetoing the beam halo and
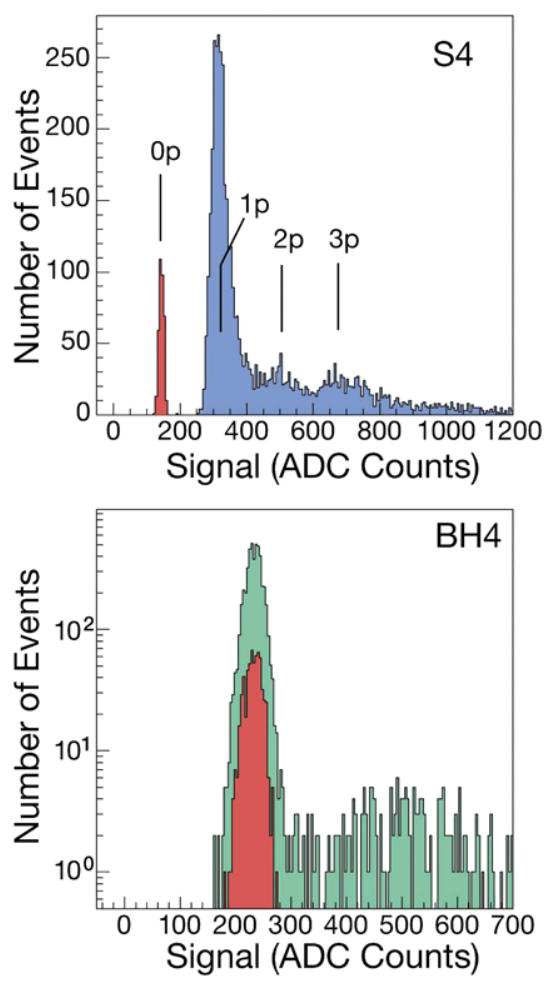

Fig. 2.5 (Color online) The signal distribution from the S4 trigger scintillator (top) for $50 \mathrm{GeV} / c$ electrons displays multi-particle events where up to three particles are clearly discernible. The bottom plot shows the signal distribution of one of the four halo counters for 3 $\mathrm{GeV} / c$ negative pion beam. The red histograms indicate pedestal distributions

large-angle particles that originated from interactions in the beam line.

\subsection{Beam composition}

In the high energy mode of the beam line, data were generally taken with negative beams. In this mode, there was no antiproton contamination. If the beam line was configured for positive particles, at very high momenta, e.g. $350 \mathrm{GeV} / c$, the beam consisted almost purely of protons. At 20 and $30 \mathrm{GeV} / c$, the proton identification in the $\pi^{+}$beam was readily possible when $\mathrm{CK} 3$ was pressurized to 1.7 bar of $\mathrm{CO}_{2}$.

The particle content depends on the momentum. At the higher end, pions dominate. At lower momenta, the beam consisted mostly of electrons. The beam consisted of $31 \%$ pions, $0.4 \%$ kaons, and $5.6 \%$ protons at $+4 \mathrm{GeV} / c$, and the remaining particles were positrons. At $+8 \mathrm{GeV} / c$, the beam contained $72 \%$ pions, $2 \%$ kaons and $7 \%$ protons, and the remaining fraction consisted of positrons. In the negatively charged beam, the particle mixture was approximately the same but the antiproton fraction was much reduced compared to that of the proton in the positive beam.

The beam content of the very low energy (VLE) beam is shown in Table 2.1. The average uncertainty on the beam 
Table 2.1 The first and second numbers in each column is the fraction of the negative and positive charged particles respectively in the VLE mode

\begin{tabular}{lllll}
\hline $\begin{array}{l}P_{b} \\
{[\mathrm{GeV} / c]}\end{array}$ & $\begin{array}{l}\left(\pi^{-}, \pi^{+}\right) \\
{[\%]}\end{array}$ & $\begin{array}{l}\left(e^{-}, e^{+}\right) \\
{[\%]}\end{array}$ & $\begin{array}{l}(\bar{p}, p) \\
{[\%]}\end{array}$ & $\begin{array}{l}\left(K^{-}, K^{+}\right) \\
{[\%]}\end{array}$ \\
\hline 9 & $73.0,68.8$ & $22.1,20.8$ & $2.7,7.6$ & $2.3,2.7$ \\
8 & $56.9,71.8$ & $39.8,19.1$ & $1.9,7.1$ & $1.4,2.1$ \\
7 & $61.8,67.2$ & $35.5,23.8$ & $1.7,7.2$ & $1.0,1.7$ \\
6 & $57.7,60.6$ & $40.1,31.3$ & $1.5,6.8$ & $0.8,1.4$ \\
5 & $53.2,51.4$ & $44.9,40.7$ & $1.2,6.6$ & $0.7,1.2$ \\
4 & $40.9,31.3$ & $58.0,62.7$ & $0.9,5.6$ & $0.2,0.4$ \\
3 & $25.9,21.9$ & $73.7,73.5$ & $0.3,4.5$ & $0.1,0.1$ \\
2 & $10.6,8.5$ & $89.3,89.2$ & $0.1,2.3$ & $0.01,0.01$ \\
\hline
\end{tabular}

fractions was $0.7 \%$ for $\pi^{-}$and electrons, and $0.4 \%$ for $\pi^{+}$ and positrons below $9 \mathrm{GeV} / c$. At $9 \mathrm{GeV} / c$, these uncertainties were $7.2 \%$ and $5.2 \%$, respectively. At beam momentum $4 \mathrm{GeV} / c$ and lower, the electrons were double tagged by CK2 and CK3 with extremely high efficiency. Above $4 \mathrm{GeV} / c, \mathrm{CK} 3$ was used to separate pions from kaons and protons. In order to enrich the hadron content of beam triggers at low momenta, a S1·S2.S4.MVF trigger was employed.

The uncertainty in pion $v s$ electron identification in general does not exceed $0.5 \%$ in the VLE mode. This uncertainty is somewhat larger, and is estimated to be $1.8 \%$ and $6 \%$ using the information from the calorimeter and Cherenkov counters at 3 and $8 \mathrm{GeV} / c$, respectively.

\section{Calibration of calorimeters}

Both the EB and HB calibrations were carried out with 50 $\mathrm{GeV} / c$ electrons. The HB calibration was performed before the EB SM was mounted in front of the HB. The electron beam was directed at the center of each tower. Similarly, the EB calibration data were collected by pointing the beam to a selected set of crystals that formed a tight grid pattern. The $r m s$ value of the linearity between 2 and $9 \mathrm{GeV} / c$ is $0.5 \%$ and less than $1 \%$ for higher energies.

The reconstructed energy was determined to be the sum of energies recorded by the EB and HB. We refer to it as the raw energy throughout this paper. For the EB, the signals from $7 \times 7$ crystals, and for the HB the signals from $3 \times 3$ towers were summed. For pion showers the $7 \times 7$ crystals contained over $99 \%$ of the energy, and the $3 \times 3$ HB towers contained over $98 \%$ of the energy. In the case of the HO, the total energy was estimated by adding signals from $3 \times 2$ towers. In each case the energy is sampled over 6 time slices $(6 \times 25 \mathrm{~ns})$ and pedestals subtracted.

The response of each $\mathrm{HB}$ scintillator tile of each layer was also measured by using a $5-\mathrm{mCi} \mathrm{Co}^{60}$ moving wire radioactive source [11]. The signal from a tower was calculated by taking the average of all measurements from the scintillator layers and weighting these averages by the shower profile. During the construction phase, every scintillator tile was tested with a radioactive wire source. This procedure makes it possible to transfer the beam calibration constants for each tower that were not placed in the test beam. The precision of tower-to-tower calibration was measured to be $2 \%$ as derived by comparing the consistency of the relative source and the beam data measurements.

Figure 3.1 shows the beam calibration constants in $\mathrm{GeV} / \mathrm{fC}$ for four $\phi$ segments as a function of $\eta$ tower numbers. The combined effect of the light attenuation in the optical fibers, loss in fiber connectors and the HPD gain differences are evident in the figure. The drop of the gain with increasing $\eta$ is due to the longer length of the optical fiber. The $\eta$ towers in a single $\phi$ segment are measured by a single HPD which has the same gain for each pixel.

At higher beam momenta ( $\geq 50 \mathrm{GeV} / c)$ the longitudinal energy leakage behind the $\mathrm{EB}+\mathrm{HB}$ is noticeable. To measure this leakage, the $\mathrm{HO}$ sampling layers were constructed and inserted in the barrel muon system to reduce these fluctuations. The HO layers (see Fig. 1.1) are placed behind the CMS solenoid cryostat. The HO system is divided into six sections that follow the division of the barrel muon system. Ring $0(+$ and -$)$ are in the central muon system and are composed of two layers of scintillators one immediately outside of the magnet cryostat and the other layer after a $15-\mathrm{cm}$ thick iron layer. Ring 0 in the muon barrel system YB0 (the central part of CMS) covers the $|\eta|$ range of 0 to 0.35 . Rings $+1,-1,+2$ and -2 are single layer scintillators inserted in the muon barrel systems YB1 and YB2 on both positive and negative sides of CMS immediately inside the first muon iron layer covering the $|\eta|$ range of 0.35 to 1.2 . In the test beam setup, the $\mathrm{HO}$ was placed behind the $\mathrm{HB}$ covering $30^{\circ}$ in $\phi$ and the full $\eta$ range. To mimic the magnet solenoid system, an aluminum block was inserted between the HB and HO. Also inserted was a $15 \mathrm{~cm}$ thick iron block between scintillator layers 1 and 2 of Ring 0 similar to the CMS detector to contain the showers. Both the aluminum

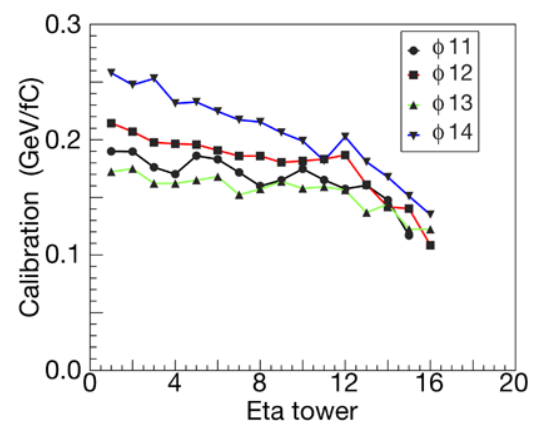

Fig. 3.1 Calibration constants for the $4 \phi$ sectors $\left(\Delta \phi=5^{\circ}\right)$ of the HB wedge used in this beam test 
and iron blocks had the same geometrical size and placement as in CMS. The $\mathrm{HO}$ modules were first calibrated by $150 \mathrm{GeV} / c \mu^{-}$beam. A clear $\mu$ peak beyond pedestal was observed in Ring 0 and Ring 2. In Ring 1 the $\mu$ peak was measurable but not as cleanly separated. Next, the HO energy scale was determined by $300 \mathrm{GeV} / c \pi^{-}$beam impinging on $\eta$ tower 4 of the HB. For this measurement, it was also required that the energy in the $\mathrm{EB}$ be less than $1.2 \mathrm{GeV}$ to constrain the energy sharing between the HB and $\mathrm{HO}$ only. The energy scale was determined by requiring the best energy resolution in $\mathrm{HB}+\mathrm{HO}$, as measured by $r m s$ width, for the $300 \mathrm{GeV} / c \pi^{-}$beam.

Figure 3.2 displays the rms energy resolution for the $\mathrm{HB}+\mathrm{HO}$ for $300 \mathrm{GeV} / c \pi^{-}$as a function of the $\mathrm{HO}$ energy scale parameter. The chosen scale parameter $(\sim 2.9)$ is the one that minimizes the combined energy resolution. Comparison of the HCAL energy spectra with and without the $\mathrm{HO}$ is shown in Fig. 3.3. It is clear that the fluctuation in energy leakage behind the $\mathrm{HB}+\mathrm{HO}$ is reduced by the introduction of the HO.

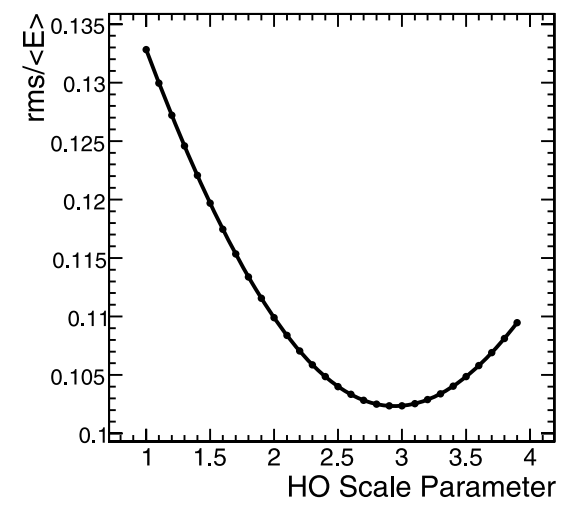

Fig. 3.2 The energy resolution of the $\mathrm{HB}+\mathrm{HO}$ response to $300 \mathrm{GeV} / \mathrm{c}$ $\pi^{-}$as a function of the $\mathrm{HO}$ scale parameter. The curve shown is a fit to the data

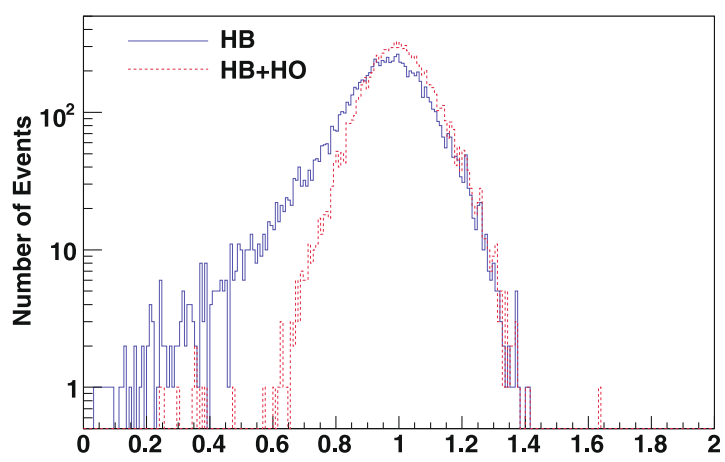

Fig. 3.3 The histograms are for the HB alone solid (blue), and for the $\mathrm{HB}+\mathrm{HO}$ dashed (red) with the optimal scale factor for the HO. The signal distributions are scaled such that $300 \mathrm{GeV} / c$ is unity

\section{Combined calorimeter $(\mathrm{EB}+\mathrm{HB})$ response}

Figure 4.1 shows the combined response of the EB $+\mathrm{HB}$ calorimeter to a variety of particles in a wide range of momenta. We define the particle response as the average calorimeter signal per unit energy, normalized to unity for electrons. Neither noise suppression nor Gaussian fitting is used in the particle response determination. At $5 \mathrm{GeV} / c$, for example, the antiproton response is $\sim 70 \%$ of the electron response. The responses to charged pions and protons are $62 \%$ and $47 \%$ of the electron response at the same energy, respectively. At a given momentum, the available energy that is converted to a calorimeter signal varies by particle type. The available energy for protons is their kinetic energy. For antiprotons, the available energy equals the kinetic energy plus twice the rest mass of the proton. For pions and kaons, the available energy is their kinetic energy plus their mass. In Fig. 4.2, the same data are plotted against the available energy, i.e. energy that contributes to the generation of an observable signal. One expects roughly the same response characteristics for all hadrons, as observed in the data, but there are subtle differences which we discuss next.

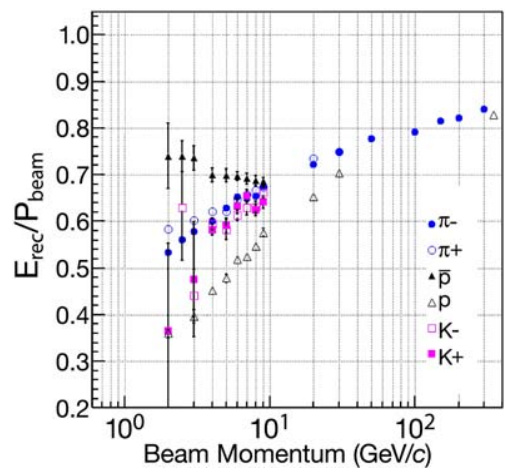

Fig. 4.1 The response of the combined calorimeter system to six different particles is shown as a function of the beam momentum. Both the $\mathrm{EB}$ and $\mathrm{HB}$ are calibrated with $50 \mathrm{GeV} / c$ electrons

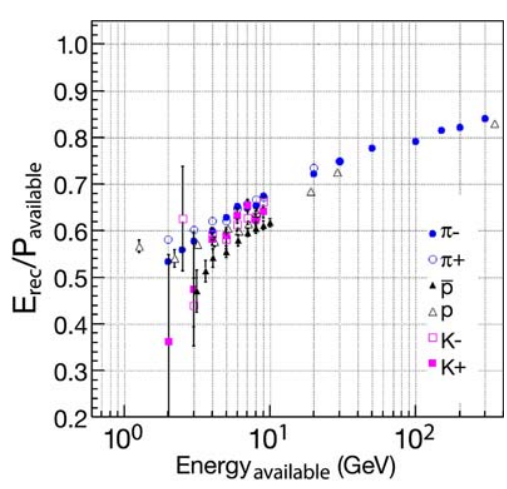

Fig. 4.2 The data are the same as in Fig. 4.1 but the calorimeter response is plotted against the available energy 


\section{$4.1\left(\pi^{+} / \pi^{-}\right)$response ratio}

The response to $\pi^{+}$below $5 \mathrm{GeV} / c$ is larger than the $\pi^{-}$ response, increasing as the energy decreases. One possible interpretation is due to the characteristics of the charge exchange reactions, $\pi^{+}+n \rightarrow \pi^{0}+p$ (I) and $\pi^{-}+p \rightarrow$ $\pi^{0}+n$ (II). $\pi^{0}$ develops electromagnetic showers which give about $20 \%$ more signal compared to hadrons. The $\pi^{0}$ production is deduced to be $10 \%$ higher at $2 \mathrm{GeV} / c$ for $\pi^{+}$ beam compared to $\pi^{-}$beam, and by $5 \mathrm{GeV} / c$, the $\pi^{0}$ is about 5\% lower [12]. Since the target material $\left(\mathrm{PbWO}_{4}\right)$ consists of about $42 \%$ more neutrons than protons, the relative effect of reaction (I) is larger than that of reaction (II). Figure 4.3 shows this effect to be about $10 \%$ at $2 \mathrm{GeV} / c$.

\section{$4.2(\pi / p)$ response ratio}

The response to protons is systematically lower than that of negative or positive pions. The most likely interpretation of this effect, also observed at high energy, is a result of the fact that $\pi^{0}$ production is, on average, smaller in proton induced showers. This is a consequence of baryon number conservation, which favors the production of leading baryons, while pion induced reactions may have leading $\pi^{0} \mathrm{~s}$. This effect was clearly observed in the HF calorimeter [13], where it caused a response difference in excess of $10 \%$. Since the $e / h$ values of the $\mathrm{EB}+\mathrm{HB}$ are smaller than for the $\mathrm{HF}^{2}$ the effects are correspondingly smaller but nevertheless significant.

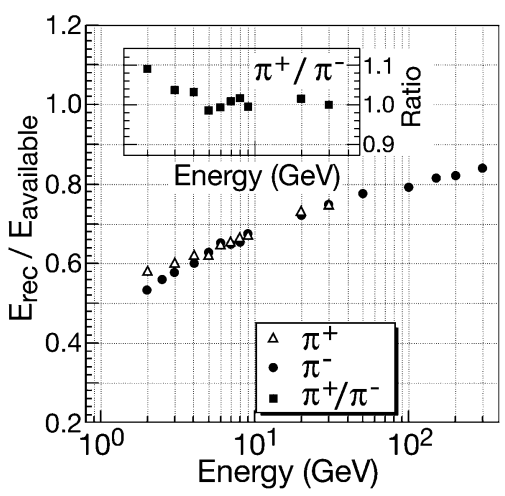

Fig. 4.3 The calorimeter response of $\pi^{+}$and $\pi^{-}$. The black squares represent the response ratio between $\pi^{+}$and $\pi^{-}$. Statistical errors are smaller than the symbol size

\footnotetext{
${ }^{2}$ The ratio of conversion efficiencies of the electromagnetic and hadronic energy depositions to electrical signals is called the intrinsic $e / h$ ratio. The ratio of responses to incident pions to incident electrons at a given energy is related to $e / h$ as " $\pi / e$ " $=\left[1+(e / h-1) f_{0}\right] /(e / h)$ where $f_{0}$ is the electromagnetic fraction, $f_{0}=0.1 \log P_{b}$, and $P_{b}$ is the beam momentum.
}

\section{$4.3(\pi / p)$ response ratio in $\mathrm{EB}$}

Since the inelastic cross sections for protons is larger than for pions, a larger fraction of the baryons start showering in the EB. This is illustrated in Fig. 4.4, which shows that $41 \%$ of the pions penetrate the EB without starting a shower, versus $35 \%$ for protons. The effective thickness of the EB is thus $1.05 \lambda_{\mathrm{I}}$ for protons and $0.89 \lambda_{\mathrm{I}}$ for pions.

Figure 4.5 shows the EB energy spectra for $300 \mathrm{GeV} / c$ negative pions and $350 \mathrm{GeV} / c$ protons. At $350 \mathrm{GeV} / c$ the positive beam is exclusively protons. The highest momentum for negative beam with sufficient intensity is about 300 $\mathrm{GeV} / c$. The ratio of noninteracting protons to pions in the $\mathrm{EB}\left(E_{\mathrm{EB}} / P_{b} \approx 0\right)$ is $\sim 1.2$ consistent with the ratio of $\lambda_{\mathrm{I}}$ as noted earlier. When these particles interact in the EB ( $E_{\mathrm{EB}} / P_{b} \approx 1$ ), the energy deposition is larger due to $\pi^{0}$ production in the case of pions compared to that of protons.

\subsection{Comparison of $\pi, p$ and $\bar{p}$ response}

The fraction of the beam energy deposited in the EB decreases from $\sim 60 \%$ at $2 \mathrm{GeV} / c$ to $\sim 25 \%$ at $300 \mathrm{GeV} / c$. At the same incident momentum, protons deposit on average less energy than pions in the EB, while antiprotons deposit more than pions. Antiprotons start their showers, on average, earlier than pions and therefore a larger fraction of the energy ends up in the EB. At first sight, one would expect

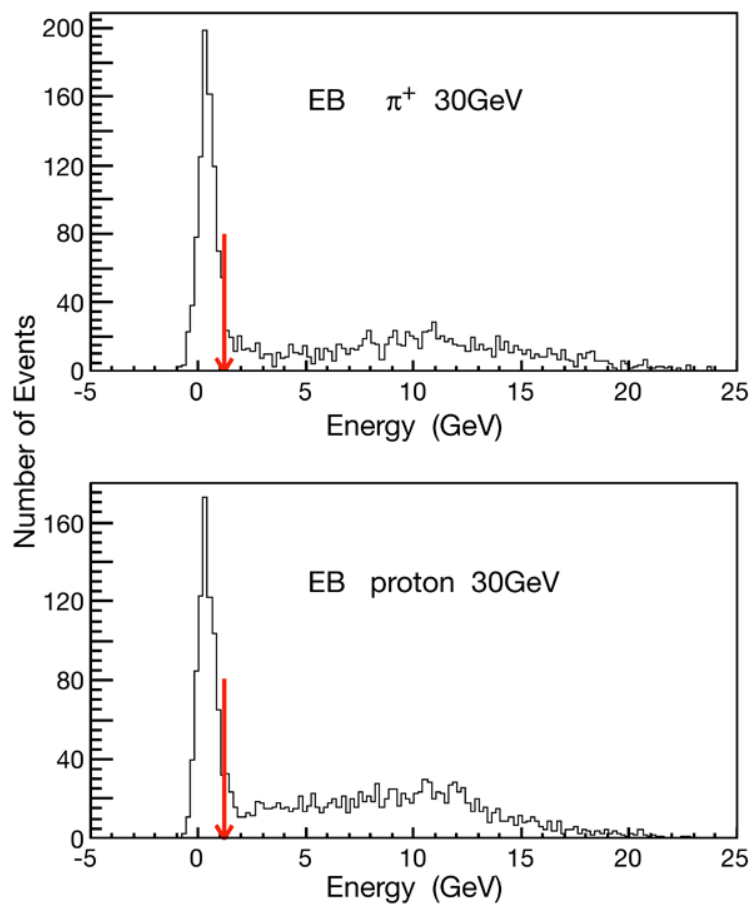

Fig. 4.4 The signal distributions for $30 \mathrm{GeV} / c$ pions (top) and protons (bottom) for the same number of events in the EB are shown. The arrow indicates where the cut is applied $(1.2 \mathrm{GeV})$ to separate noninteracting pions and protons from the interacting ones 


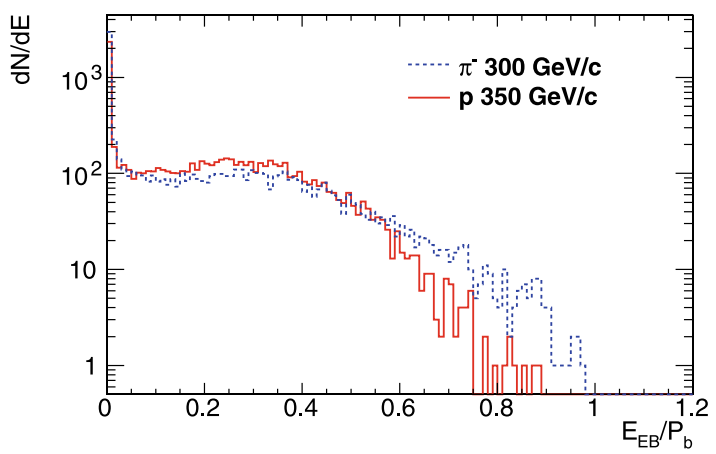

Fig. 4.5 The EB energy distribution for $300 \mathrm{GeV} / c$ pions and $350 \mathrm{GeV} / c$ protons. The two histograms contain the same number of entries and the horizontal axis is normalized by the beam momentum

the same for proton induced showers. However, when a proton interacts in the EB, the interactions have limited energy transfered to secondary $\pi^{0} \mathrm{~s}$ because the final state should contain two baryons. This effect suppresses the proton signal in the EB, despite the fact that protons are more likely to start their showers in the EB compared to pions. The requirements of baryon number conservation do not limit $\pi^{0}$ production for antiproton induced showers. In first approximation, there is no difference with pion induced showers.

The effects mentioned above also explain why the antiproton response is systematically smaller that the pion response (Fig. 4.1). Antiprotons are more likely to start showering in the EB compared to the pions. Pions deposit, on average a larger fraction of their energy in the HB. And since the $e / h$ value of the $\mathrm{HB}$ is smaller than for the $\mathrm{EB}$, the pions benefit more from the increased response to the nonelectromagnetic shower components.

\section{$4.5 \mu$ response}

Figure 4.6 shows the response of $150 \mathrm{GeV} / \mathrm{c}$ muons in the HB using $3 \times 3$ HB tower structure. Even though 9 towers were read, only the central tower has a signal above pedestal. Since the noise in a single tower of the HB is equivalent to

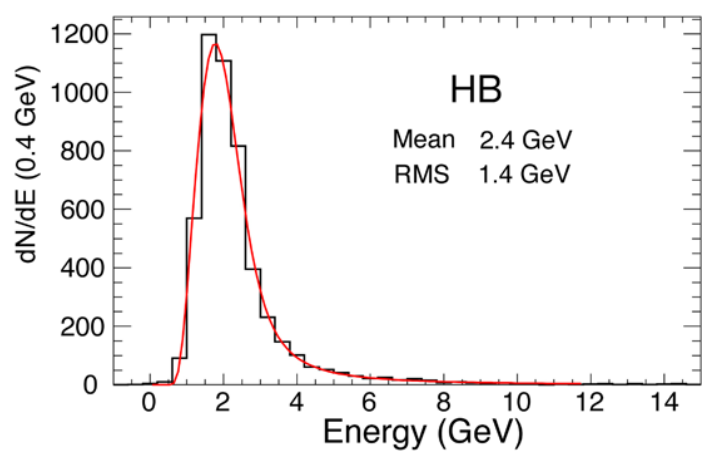

Fig. 4.6 The HB signal distribution for $150 \mathrm{GeV} / c \mu^{-}$from tower 4 $(\eta=0.3)$. The solid curve represents a fit using combined Gaussian and Landau distributions $\sim 0.2 \mathrm{GeV}$, this calorimeter system is superb in identifying single isolated muons. The HB trigger electronics is also designed to generate an isolated muon signal (bit) based on this capability. Muons can also be used as a relative calibration of every tower. Using the $50 \mathrm{GeV} / c$ electron calibration, the mean energy deposited by a $150 \mathrm{GeV} / c$ muon is $2.4 \pm 0.1$ $\mathrm{GeV}$. If the pion calibration correction is applied, the mean energy deposited is at $2.8 \pm 0.2 \mathrm{GeV}$.

\section{Optimization of energy reconstruction}

The total energy in the CMS central calorimeter system is the sum of signals from the EB, HB and HO. The $e / h$ values are very different for the EB and the HB, and thus corrections have to be applied to obtain the true particle energy from the combined system.

Figure 5.1 displays the measured energy correlations in the EB $v s$ HB towers for a number of pion beams. In a compensating calorimeter $(e / h=1)$, the events would lay about the solid lines as indicated in Fig. 5.1. This is not the case for the $\mathrm{EB}+\mathrm{HB}$ system, and thus we perform optimization of the energy response in three steps using the cluster energies from $7 \times 7$ EB crystals, $3 \times 3 \mathrm{HB}$ and $3 \times 2 \mathrm{HO}$ towers. We consider energies at least $3 \sigma$ away from the noise level $(0.8$, 1.0 and $2.0 \mathrm{GeV}$ for the $\mathrm{EB}, \mathrm{HB}$ and $\mathrm{HO}$, respectively). In this section, the measured energy always refers to the cluster energy unless specified otherwise. The first correction is carried out for the HB energy using minimally ionizing events in the EB. The next step utilizes the corrected energies in the HB and the beam constraint to correct the energy measured by the EB. It is important to note that the usage of the known beam momentum at this point is only to determine the parametrization. Finally, using the corrected EB and HB energies from the above steps, the nonlinear response of the combined EB $+\mathrm{HB}$ system as a function of the EB energy fraction is described using a third order polynomial.

The first task is to parametrize the $\pi / e$ ratio for the HB and we use events that deposit minimum ionizing energy in the $\mathrm{EB}\left(E_{\mathrm{EB}}<1.2 \mathrm{GeV}\right)$. Figure 5.2 displays the mean of $\pi / e$ as a function of the measured energy in the HB. The plot (in semi-log) shows two lines with a break point at about $8 \mathrm{GeV}$. Above $\sim 8 \mathrm{GeV}$, the mean of $\pi / e$ can be expressed, for example, by a fit using Wigmans' parametrization $[14,15]$ with $e / h=1.4 \pm 0.1$,

$\left\langle(\pi / e)_{\mathrm{HB}}\right\rangle=\frac{1+(e / h-1) \times 0.1 \log \left(E_{\mathrm{HB}}\right)}{e / h}$.

Below $\sim 8 \mathrm{GeV}, \pi / e$ is represented by the following logarithmic function

$$
\left\langle(\pi / e)_{\mathrm{HB}}\right\rangle=0.179 \pm 0.005 \log \left(E_{\mathrm{HB}}\right)+0.413 \pm 0.005 .
$$


Fig. 5.1 The raw energy deposit correlations between the EB and HB for 300, 100, 20, 8, 4 and $2 \mathrm{GeV} / c \pi^{-}$s. The straight line indicates the behavior of a compensating calorimeter system
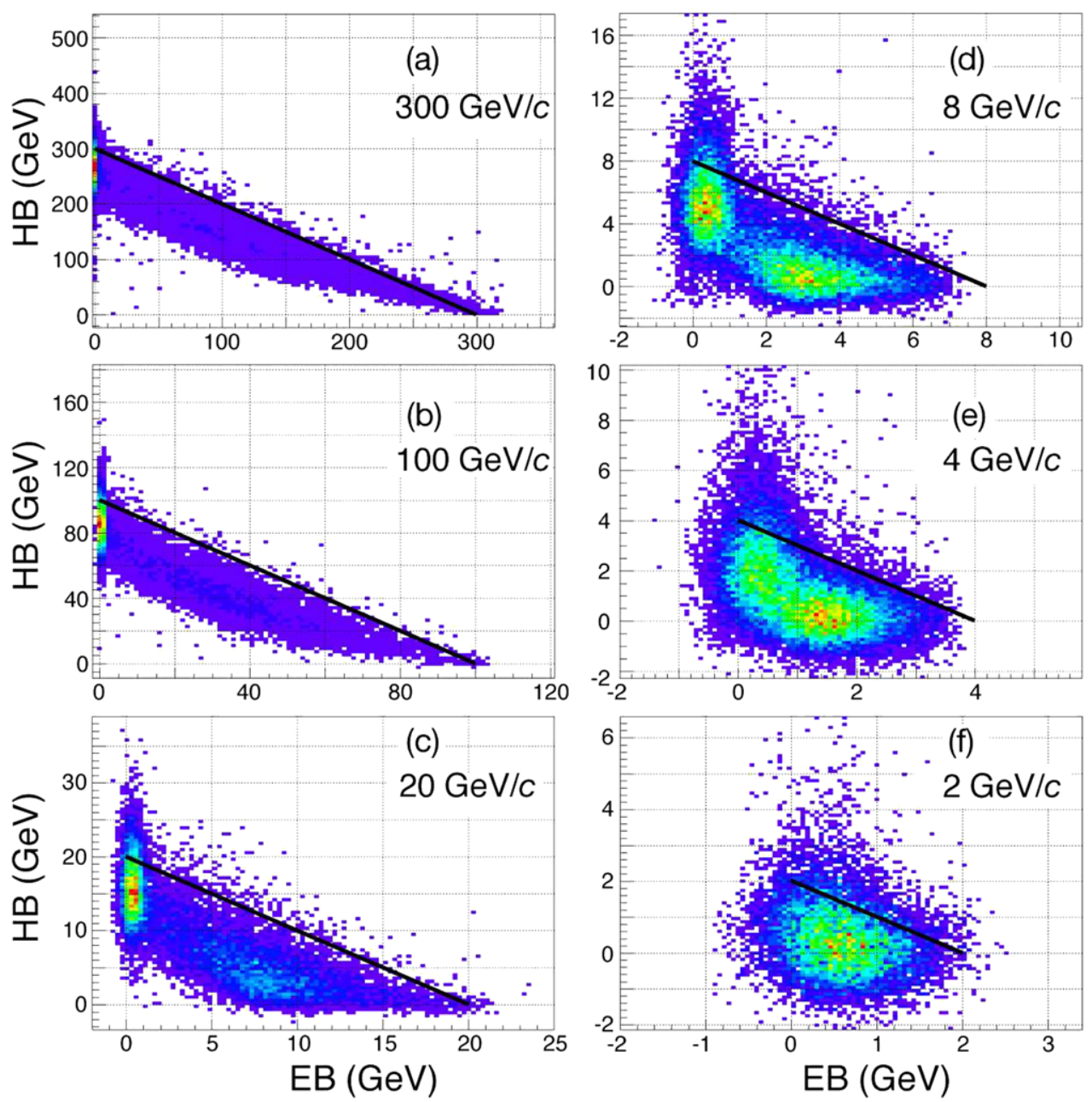

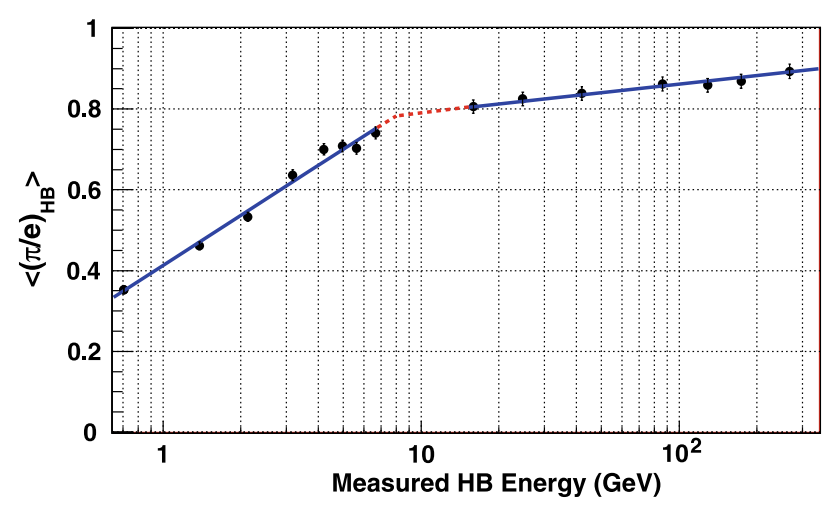

Fig. 5.2 $\pi / e$ vs $E_{\mathrm{HB}}$ for events interacting in the HB. The data are fit to two separate log functions with a break at about $8 \mathrm{GeV}$

Another approach is due to Groom [16]: $\pi / e=1-(1-$ $h / e)\left(E / E_{0}\right)^{m-1}$ where $E_{0}$ is about $1 \mathrm{GeV}$ and $m$ ranges from 0.80 to 0.85 . A fit to the data above $20 \mathrm{GeV}$ without fixing $E_{0}$ and $m$ gives $e / h=1.5, E_{0}=2.5 \mathrm{GeV}$ and $m=$ 0.77. Neither Wigmans nor Groom parametrization works well for the entire energy range from 2 to $300 \mathrm{GeV}$.
The next step is to correct the energy deposited in the EB using the event-by-event corrected energy values in the HB using Eqs. 1 and 2. The EB energy is simply the beam energy minus the corrected energy in the HB. The ratio of the computed EB energy divided by the measured energy is $\pi / e$ (Eq. 3). In order to determine the EB correction parameters in Eq. 4, we require sizable energy both in the EB and HB. For each beam momentum, the average EB energy is computed by using the following formula:

$\left\langle(\pi / e)_{\mathrm{EB}}\right\rangle=\frac{\left\langle E_{\mathrm{EB}}\right\rangle}{P_{b}-E_{\mathrm{HB}}^{*}}$

where $E_{\mathrm{HB}}^{*}$ is the event-by-event corrected $\mathrm{HB}$ energy, $E_{\mathrm{HB}} /(\pi / e)_{\mathrm{HB}}$. In Fig. 5.3, the mean $\pi / e$ for EB is plotted $v s$ the logarithm of the observed mean EB energy. The line is fitted to the data with a function of the form

$\left\langle(\pi / e)_{\mathrm{EB}}\right\rangle=a_{\mathrm{E}} \log \left(E_{\mathrm{EB}}\right)+b_{\mathrm{E}}$.

The best fit parameters are $a_{\mathrm{E}}=0.057 \pm 0.006$ and $b_{\mathrm{E}}=$ $0.49 \pm 0.04$. 
After correcting the EB energies event-by-event using the above function, $E_{\mathrm{EB}}^{*}=E_{\mathrm{EB}} /(\pi / e)_{\mathrm{EB}}$, we find that the $\pi / e$ correction overestimates the total $\mathrm{EB}+\mathrm{HB}$ energy values for events with large $\mathrm{EB}$ energy fractions, $Z \equiv E_{\mathrm{EB}} /\left(E_{\mathrm{EB}}+\right.$ $\left.E_{\mathrm{HB}}\right)>70 \%$ (see Fig. 5.4). This is expected since these events correspond to the cases when a hadronic shower in the EB fluctuates largely to neutral particles. The final step in the correction sequence is to parametrize the nonlinear response of the combined system with a function as indicated in Fig. 5.4. This set of corrections has been determined to be insensitive to the beam momentum and $100 \mathrm{GeV} / c$ data is a good representation for all other beam momentum data.

$$
\begin{aligned}
\left\langle\frac{E_{\mathrm{EB}}^{*}+E_{\mathrm{HB}}^{*}}{P_{b}}\right\rangle= & (0.412 \pm 0.045) Z^{3}-(0.096 \pm 0.058) Z^{2} \\
& -(0.084 \pm 0.018) Z+1.00
\end{aligned}
$$

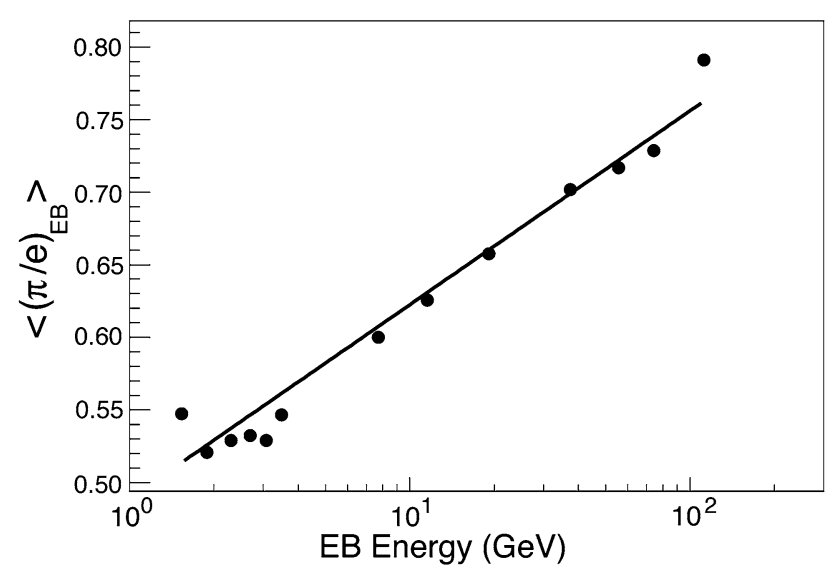

Fig. 5.3 Measured $(\pi / e)_{\mathrm{EB}} v s E_{\mathrm{EB}}$ after correcting the energies of pions that interacted in the EB (see text for details)

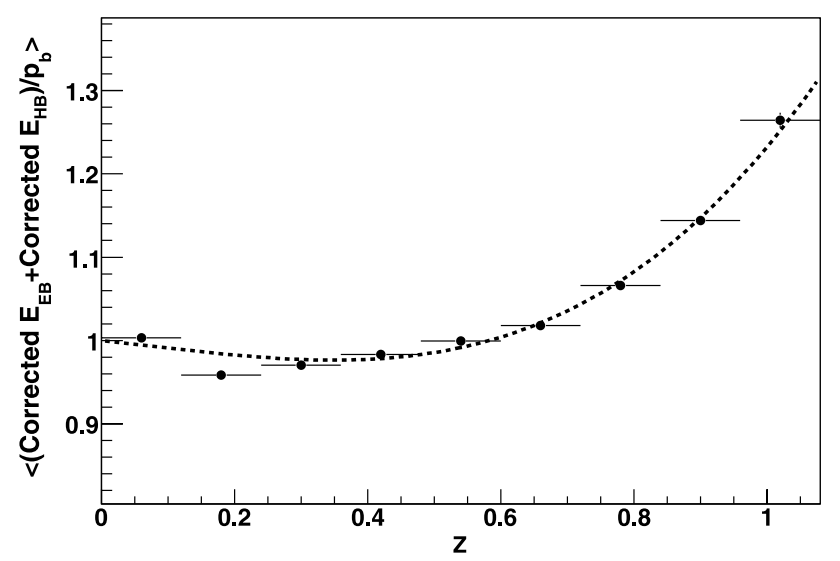

Fig. 5.4 The $\pi / e$ corrected response ratio for $100 \mathrm{GeV} / c$ pions of the combined system as a function of the EB fraction. The $Z$ value is defined as $E_{\mathrm{EB}} /\left(E_{\mathrm{EB}}+E_{\mathrm{HB}}\right)$, ratio of raw energy deposit in the $\mathrm{EB}$ with respect to the total in the calorimeter. The smooth curve is a third order polynomial fit to the data (see Eq. 5)
The total response of the $\mathrm{EB}+\mathrm{HB}$ system can be optimized by applying Eqs. 1, 2, 4, and 5 event-by-event. These event-by-event corrected EB vs HB energy values are shown in Fig. 5.5 (e.g. 20 and $100 \mathrm{GeV} / c$ ). The improvement in the nonlinear behavior and the response with respect to the distributions in Fig. 5.1 is clearly visible in this figure. Figure 5.6 shows the signal distributions at four beam momenta before and after the corrections. Corrections bring the mean of these distributions to the corresponding beam momentum value.

In order to calculate the energy resolution of the combined $\mathrm{EB}+\mathrm{HB}$ system, the mean and $r m s$ values were computed for each momentum. For $5 \mathrm{GeV} / c$ data and above, a Gaussian fit was also performed for the raw and the corrected data. Below $5 \mathrm{GeV} / c$, the signal distributions deviate from Gaussian distribution substantially and were not included in the energy resolution determination. Moreover, the correction method did not help 2 and $3 \mathrm{GeV} / c$ data. Figure 5.7 displays the energy resolution and the response of the combined $\mathrm{EB}+\mathrm{HB}$ calorimeters for pions. The circles represent the raw and the squares represent the corrected data. Figures 5.7a and $\mathrm{b}$ are derived from sample means and the rms values, whereas Figs. 5.7c and d are constructed using the Gaussian fit values to the corresponding energy distributions. The energy resolution is customarily parametrized as $\sigma / E=a / \sqrt{E} \oplus b$ where $a$ is the stochastic and $b$ is the constant term, and the terms are added in quadrature. The raw resolution of the $\mathrm{EB}+\mathrm{HB}$ system is such that $a=111.5 \pm 2.1 \%$ and $b=8.6 \pm 1.4 \%$ as indicated by open black circles within 4 to $300 \mathrm{GeV} / \mathrm{c}$ in Fig. 5.7a. After applying the corrections, the energy resolution improves,
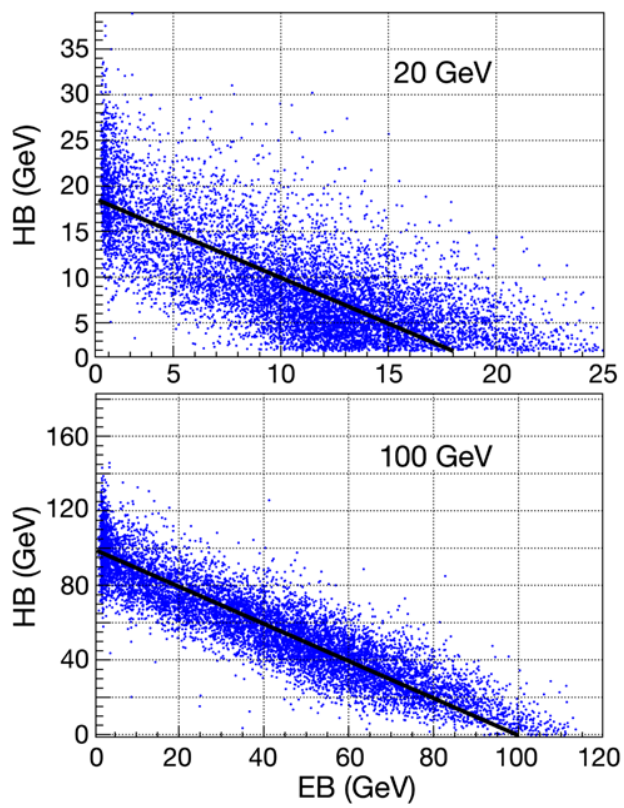

Fig. 5.5 The EB $v s \mathrm{HB}$ energy for incident pions of 20 and $100 \mathrm{GeV} / c$ after the correction steps described in the text are applied 

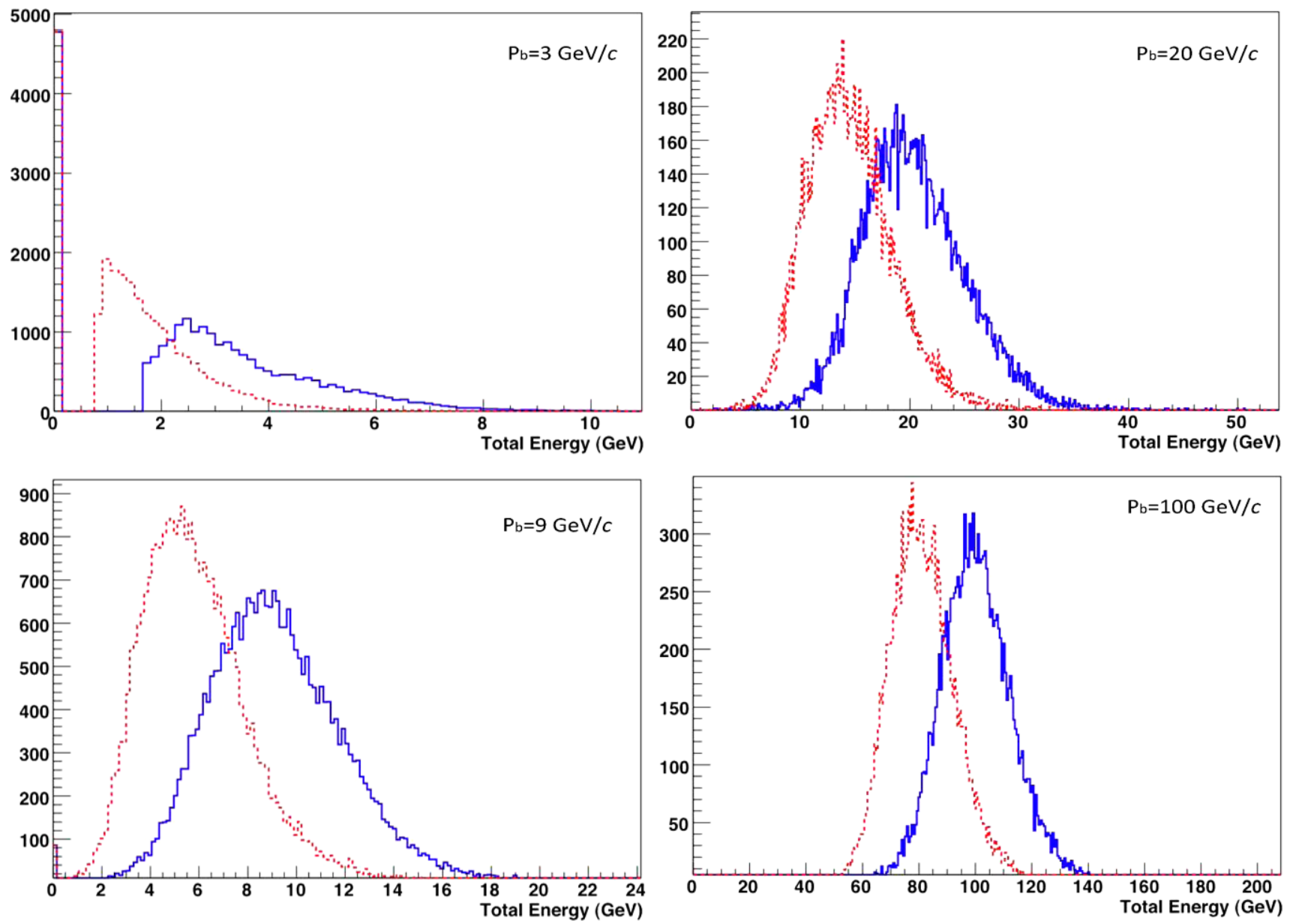

Fig. 5.6 The signal distributions at four incident $\pi^{-}$beam momenta. The dashed (red) histograms are the raw data and the solid (blue) histograms are after the corrections described in the text

Fig. 5.7 The energy resolution (a and c) and the corrected response of the combined calorimeters (b and $\mathbf{d}$ ) before (circles) and after the corrections (squares) are discussed in detail in the text
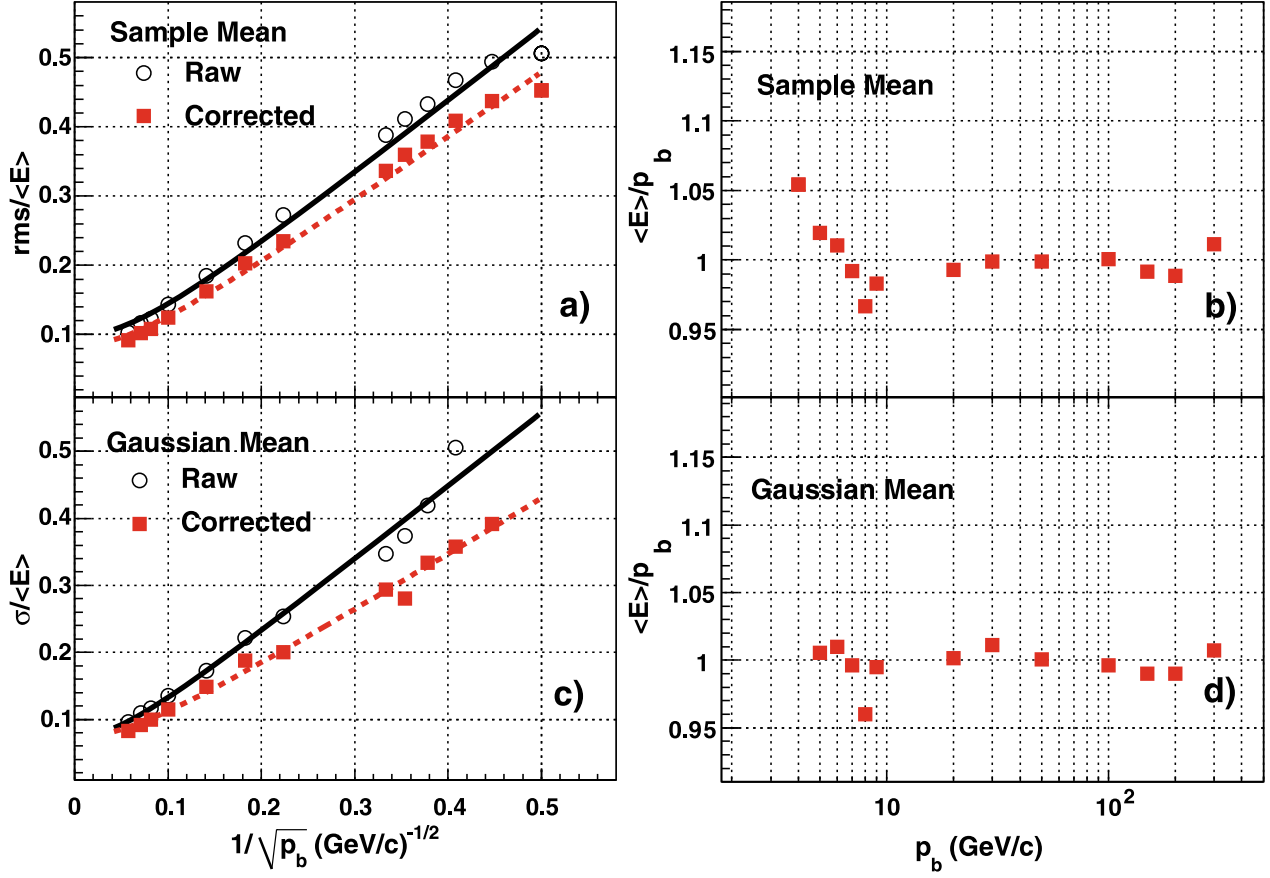
$a=94.3 \pm 1.2 \%$ and $b=8.4 \pm 1.0 \%$ (solid red squares in Fig. 5.7a). The open black circles in Fig. 5.7c display the energy resolution of the combined system when the raw data are fit with a Gaussian distribution at each energy from 5 to $300 \mathrm{GeV} / c$. This procedure results in $a=110.7 \pm 3.1 \%$ and $b=7.3 \pm 1.7 \%$. The corrections further improve the energy resolution as indicated by the solid red squares in Fig. 5.7c ( $a=84.7 \pm 1.6 \%$ and $b=7.4 \pm 0.8 \%$ ). The corrected mean response remains constant within $1.3 \% \mathrm{rms}$ as depicted in Fig. 5.7d.

The method described in this section was developed to improve the total response of the isolated charged hadron clusters in the $\mathrm{EB}+\mathrm{HB}$. The application of the method (i.e., Eqs. 1, 2, 4, and 5) requires only the measured cluster energy values in the EB and $\mathrm{HB}$ for each event and it may be further improved by taking into account the transverse energy distribution information in the EB and HB.

\section{Summary and conclusions}

The CMS barrel calorimeter has been exposed to particle beams with momenta from 2 to $350 \mathrm{GeV} / c$. The beam line instrumentation included Cherenkov, time-of-flight, and veto counters, as well as wire chambers. The particle identification was sufficient to separate electrons, muons, pions, kaons and protons over a substantial energy range. At the higher energies, the CMS outer hadron calorimeter, the HO, was employed to reduce the fluctuations in longitudinal energy leakage.

The response to different hadrons is examined and interesting regularities have emerged. The ratio of negative to positive charged pion response, the ratio of negative pion to proton response and the ratio of pions to antiprotons are explored.

Finally, the linearity and energy resolution for negative pions are optimized. The CMS calorimetry is noncompensating and the EB and $\mathrm{HB}$ segments are of disparate materials. Thus, the raw response and the energy resolution need to be corrected. In particular, the present data set explores the low energy (below $10 \mathrm{GeV}$ ) response where previously used parametrizations no longer fit the data well. Since this is precisely the relevant energy regime for many of the particles in jets, it is important to understand and develop correction strategies for these particles.

The $\pi / e$ ratio of both the $\mathrm{EB}$ and $\mathrm{HB}$ is fit over 5$300 \mathrm{GeV} / c$ range. The corrected data are linear within $1.3 \%$ (rms) above $5 \mathrm{GeV} / c$, and the stochastic and the constant energy resolution terms are $84.7 \pm 1.6 \%$ and $7.4 \pm 0.8 \%$, respectively. The calorimeter remains noncompensating, so that a substantial deviation from $E^{-1 / 2}$ scaling is unavoidable. The correction method outlined in this paper is for isolated single particles. The experimental data however can be applied to jets with the aid of Monte Carlo techniques where the jet response is reconstructed from known individual particles studied in these tests.

Acknowledgements The results presented in this paper are partially based on the doctoral theses of Jordan Damgov [17], Kazim Gümüş [18] and Efe Yazgan [19]. This project was carried out with financial support from CERN, Department of Atomic Energy and Department of Science and Technology of India, U.S. Department of Energy, U.S. National Science Foundation, RMKI-KFKI (Hungary, OTKA grant T 016823), Croatian Ministry of Science, Education and Sport (under grant No. 023-0982887-3064), French CNRS/Institut de Physique Nucleaire et de Physique des Particules, French Commissariat a l'Energie Atomique, Greek General Secretariat for Research and Technology, Italian Istituto Nazionale di Fisica Nucleare, Federal Agency for Science and Innovations of the Ministry for Education and Science of the Russian Federation, Federal Agency for Atomic Energy of the Russian Federation, Russian Academy of Sciences, Ministry of Science of Serbia, Swiss Funding Agencies, Scientific and Technical Research Council of Turkey (TÜBITAK), Turkish Atomic Energy Agency (TAEK), Bogazici University Research Fund (Grant no: 04B301), Science and Technology Facilities Council (UK).

\section{References}

1. CMS Collaboration, The hadron calorimeter project technical design report, CERN/LHCC 97-31, 1997

2. CMS Collaboration, The electromagnetic calorimeter technical design report, CERN/LHCC 97-33, 1997

3. B.S. Archaya et al., The CMS outer calorimeter, CMS NOTE2006/127

4. S. Abdullin et al., Eur. Phys. J. C 53, 139 (2008)

5. S. Abdullin et al., Eur. Phys. J. C 55, 159 (2008)

6. V. Abramov et al., Nucl. Instrum. Methods A 457, 75 (2001)

7. G. Baiatian et al., Energy response and longitudinal shower profiles measured in CMS HCAL and comparison with Geant4, CMS NOTE-2006/143

8. G. Baiatian et al., Synchronization and timing in CMS HCAL, CMS NOTE-2006/139

9. W. Bertl et al., Eur. Phys. J. C 41, 11 (2005)

10. P. Adzic et al., Eur. Phys. J. C 44, 1 (2006)

11. M. Adams et al., Nucl. Instrum. Methods A 511, 311 (2003)

12. C. Amsler et al., Phys. Lett. B 667, 1 (2008)

13. N. Akchurin et al., Nucl. Instrum. Methods A 408, 380 (1998)

14. R. Wigmans, Nucl. Instrum. Methods A 265, 273 (1988)

15. C.W. Fabjan, T. Ludlam, Annu. Rev. Nucl. Part. Sci. 32, 335 (1982)

16. T.A. Gabriel et al., Nucl. Instrum. Methods A 338, 336 (1994)

17. J. Damgov, $\mathrm{PhD}$ thesis, Institute for Nuclear Research and Nuclear Energy, Bulgarian Academy of Science, Sofia, Bulgaria (unpublished), 2008

18. K. Gümüş, PhD thesis, Texas Tech University, CERN-THESIS2008-066

19. E. Yazgan, $\mathrm{PhD}$ thesis, Middle East Technical University, Ankara, Turkey, Fermilab-thesis-2007-13 